\title{
On corepresentations of one parametric equipped posets
}

\author{
Claudio Rodríguez Beltrán \\ Universidad Nacional de Colombia, Sede Bogotá \\ E-mail address: crodriguezbe@unal.edu.co
}

\begin{abstract}
Corepresentations of some one parametric equipped posets over an arbitrary quadratic field extension $\mathbb{F} \subset \mathbb{G}$ are studied and classified. Complete matrix canonical forms of indecomposable corepresentations of several sincere one parametric equipped posets are presented, including those for two critical equipped posets. Some important properties of corepresentations of equipped posets are established.
\end{abstract}

\section{Introduction}

Soon after the time when the interest for studying representations of algebras by using quiver methods arose, it came the idea to investigate representations of quivers with additional structures. For instance, Dlab and Ringel considered representations of valued graphs [6]-[8], Nazarova and Roiter introduced representations of polyquivers [15] the theory of which was then developed in [16], [17].

Later, during the 90's, an analogous interest for investigating representations of posets with additional structures was realized in several directions. On the one hand, Klemp and Simson studied representations of Schurian vector space categories and considered closely related with them representations of valued posets [12]. On the other hand,

Received by the editors February 11 of 2010.

Partially supported by the Fundación para la Promoción de la Investigación y la Tecnología del Banco de la Republica de Colombia, Project 2.402. 
Zabarilo and Zavadskij [22] introduced and started to investigate representations of equipped posets over the classical pair of fields $(\mathbb{R}, \mathbb{C})$, with further developing of this theory in $[23],[24]$.

Recently, in [20], there were introduced corepresentations of equipped posets over an arbitrary quadratic field extension $\mathbb{F} \subset \mathbb{G}$. They led to some matrix problems of mixed type (over a pair of fields) which are similar to the problems of the representation case. Moreover, in some intuitive sense, the appeared problems are dual to the problems of the representation case. Nevertheless it is not known a formal construction reducing one kind of the problems to another one.

The aim of this paper is to investigate and classify corepresentations of some equipped posets of infinite type, mainly one parametric. Notice that the finite type case was solved in fact in [12], both for representations and corepresentations.

We consider several sincere one parametric equipped posets including two critical ( $K_{6}, K_{8}, A_{25}, A_{28}, A_{38}, A_{41}$ and their duals, in the notation of [24]) and obtain for them an evident and complete matrix classification of indecomposable corepresentations over the pair $(\mathbb{F}, \mathbb{G})$. Also, we establish some properties of equipped posets of infinite type (not necessarily of one parameter type) containing a well inserted critical subposet $K_{6}$ or $K_{8}$ (see the definitions of Sections 3.1 and 3.2).

It should be mentioned that analogous properties, which involve some well inserted critical subposets, have played an important role in the classical representation theory of ordinary posets [2], [28], as well as in the study of representations of equipped posets [22]. The properties obtained in the present paper are supposed to be used subsequently to get a complete classification of corepresentations of all one parametric equipped posets.

Our investigation may be considered as a natural continuation and extension of the previous developments in the one parametric representation case realized in [19], [3], [22].

The structure of this paper is the following. Section 2 contains basics definitions and notations on corepresentations of equipped posets.

The main results, describing the properties of well inserted subposets and the classification of corepresentations of some critical equipped posets, are formulated in Section 3 (see Theorems 3.1, 3.3, 3.5, 3.6 and Corollaries 3.2, 3.4). 
Section 4 provides the proofs of the Theorems 3.5 and 3.6 concerning the classification of corepresentations of the critical sets $K_{6}$, $K_{8}$, as well as several classification results on corepresentations of the equipped posets $A_{25}, A_{28}, A_{38}, A_{41}$.

In Section 5, Theorems 3.1 and 3.3 (describing the mentioned properties of critical well inserted posets) are proved.

In Section 6, we present the notations for the appendixes.

The obtained canonical matrix forms of the classified corepresentations are shown in Appendixes A-D. Appendix E contains a table with the roots of some equipped posets along with the reflections of which they came from, see Section 3.3.

\section{Main Definitions and Notations}

We briefly recall here the main definitions and notations on corepresentations of equipped posets, both introduced in [20] and some new ones. As for representations of equipped posets, the reader may consult [22]-[24] and especially [20] (the last presentation is the most close to the exposition here).

2.1. Equipped posets. An equipped poset is a triple (P) $\leq, \triangleleft)$ where $(\mathcal{P}, \leq)$ is a poset and $\triangleleft$ is an additional binary relation on $\mathcal{P}$, called strong such that the following condition holds

$$
x \leq y \triangleleft z \quad \text { or } \quad x \triangleleft y \leq z \quad \text { implies } \quad x \triangleleft z,
$$

i.e. a composition of a strong relation $\triangleleft$ with any other relation is strong (our notation $\triangleleft$ corresponds to $\unlhd$ in [20]).

We write $x \prec y$ if $x \leq y$ and $x \nless y$ and call the relation $\prec$ weak. Notice that in general neither $\prec$, nor $\triangleleft$ is partially order relation on $\mathcal{P}$ (they could be not reflexive).

For the brevity, we will write simply $\mathcal{P}$ instead of $(\mathcal{P}, \leq, \triangleleft)$. All the considered posets are finite.

A point $x \in \mathcal{P}$ is called strong (weak) if $x \triangleleft x(x \prec x)$, with the notation in diagrams $\circ(\otimes)$. By $\mathcal{P}^{\circ}\left(\mathcal{P}^{\otimes}\right)$ we denote the subset of all strong (weak) points of $\mathcal{P}$. If $\mathcal{P}=\mathcal{P}^{\circ}$, the equipment is trivial and the poset $\mathcal{P}$ is ordinary. Each subset $X \subset \mathcal{P}$ consisting of strong points only is said to be ordinary. 
We write $x \varangle y$ if the points are incomparable. For any subset $X \subset \mathcal{P}$ denote

$$
N(X)=\{a \in \mathcal{P}: a æ x \text { for all } x \in X\} .
$$

Remark 1. From (2.1) it follows that, for any weak relation $x \prec y$, both points $x$ and $y$ are weak, moreover, for any point $x \leq t \leq y$ it holds $x \prec t \prec y$.

Given some subsets $X_{1}, \ldots, X_{n} \subset \mathcal{P}$ (which may have comparable elements in different subsets), we denote by $X_{1}+\cdots+X_{n}$ their disjoint union and we call it the sum of $X_{1}, \ldots, X_{n}$. Let $|X|$ be the cardinality of a set $X$. Sometimes we identify a one-point subset $\{x\} \subset \mathcal{P}$ with the point $x$ itself.

For a point $x \in \mathcal{P}$, denote $x^{\vee}=\{y: x \leq y\}, x^{\nabla}=\{y: x \triangleleft y\}$ and $x^{\curlyvee}=\{y: x \prec y\}$. Notice that $x^{\vee}$ and $x^{\nabla}$ are upper cones meanwhile $x^{\curlyvee}$ is not in general. The subsets $x_{\wedge}, x_{\Delta}, x_{\curlywedge}$ are dually defined.

For a subset $X \subset \mathcal{P}$, set $X^{\Re}=\bigcup_{x \in X} x^{\Re}$ where $\Re$ is any of the symbols $\vee, \wedge, \nabla, \Delta, \curlyvee, \curlywedge$. We say that some relation $x R y$ between two points is strict if $x \neq y$. For subsets $X, Y \subset \mathcal{P}, X \leq Y$ means $x \leq y$ for all $x \in X$ and $y \in Y, X \prec Y$ and $X \triangleleft Y$ are analogously defined.

A subset of $\mathcal{P}$ is a chain (anti-chain) if all its points are pairwise comparable (incomparable), in particular, a dyad (triad) is an antichain of two (three) points. The length of a chain is the number of its points. A chain of the form $a_{1} \prec a_{2} \prec \cdots \prec a_{n}$ is called weak, if additionally $a_{1} \prec a_{n}$ then it is completely weak. An arbitrary subset $X \subset \mathcal{P}$ is said to be completely weak if all its points and possible relations between them are weak.

By a subposet of an equipped poset $\mathcal{P}$ we understand any of its full equipped subset $\mathcal{Q} \subset \mathcal{P}$, which means that for any two points $x, y \in \mathcal{Q}$ it holds $x \prec y(x \triangleleft y)$ in $\mathcal{Q}$ if and only if $x \prec y(x \triangleleft y)$ in $\mathcal{P}$.

Graphically each equipped poset is presented by its diagram, which is obtained from the ordinary Hasse diagram of the poset $\mathcal{P}$ by distinguishing its strong and weak points $(o$ and $\otimes)$ and by joining additional lines symbolizing those strong strict relations between weak points which are not consequences of other relations.

Example 2.1. Let $\mathcal{P}$ be an equipped poset given by the diagram of Figure 1 . Then, among strict relations, the only weak ones are $1 \prec$ $\{2,5\}, \quad\{2,5\} \prec 3$, hence all those in the rest are strong, namely, $1 \triangleleft 3, \quad\{1,2,3,5, b\} \triangleleft 4, \quad\{1,2\} \triangleleft a, \quad\{1,5, b\} \triangleleft c, \quad b \triangleleft\{3,4,5, c\}$. 


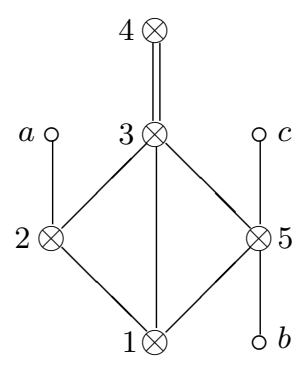

FIGURE 1. Equipped poset.

Remark 2. Notice that, in the analogous Example 2.2 in [20], the additional arc joining the points 1 and 3 is superfluous and must be omitted (in that example $1 \triangleleft 3$ is a consequence of $1 \triangleleft 5 \prec 3$ ).

2.2. The category of corepresentations. We recall now the main information on corepresentation category following [20]. Let $\mathbb{F} \subset$ $\mathbb{F}(\xi)=\mathbb{G}$ be an arbitrary quadratic field extension, so, each $x$ in $\mathbb{G}$ is expressed in unique form $x=a+\xi b(a, b \in \mathbb{F})$ where $a=\operatorname{Re}(x)$ is said to be the real part of $x$ and $b=\operatorname{Im}(x)$ the imaginary part of $x$. Each equipped poset $\mathcal{P}$ naturally defines a matrix problem of mixed type over the pair $(\mathbb{F}, \mathbb{G})$.

Consider a rectangular matrix $M$ over $\mathbb{G}$ separated into vertical stripes $M_{x}, x \in \mathcal{P}$ :

$$
M=\begin{array}{l|l|l|l|l|}
\multicolumn{4}{c}{x} & \multicolumn{1}{c}{y} \\
\hline \ldots & M_{x} & \ldots & M_{y} & \ldots \\
\hline
\end{array}
$$

such partitioned matrices $M$ are called matrix corepresentations of $\mathcal{P}$ over $(\mathbb{F}, \mathbb{G})$. Their admissible transformations are the following:

(a) $\mathbb{G}$-elementary row transformations of the whole matrix $M$;

(b) $\mathbb{G}$-elementary ( $\mathbb{F}$-elementary) column transformations of a stripe $M_{x}$ if the point $x$ is strong (weak);

(c) In the case of a strong (weak) relation $x \triangleleft y(x \prec y)$, additions of columns of the stripe $M_{x}$ to the columns of the stripe $M_{y}$ with coefficients in $\mathbb{G}(\mathbb{F})$. 
Two corepresentations are said to be equivalent or isomorphic if they can be turned into each other with help of the admissible transformations. The corresponding matrix problem of mixed type over the pair $(\mathbb{F}, \mathbb{G})$ consists in classifying the indecomposable corepresentations in the natural matrix sense, up to equivalence.

To every matrix corepresentation $M$ is associated the vector dimension $d=\underline{\operatorname{dim}} M=\left(d_{0}, d_{x}: x \in \mathcal{P}\right)$ where $d_{0}\left(d_{x}\right)$ is the number of rows in $M$ (of columns in $M_{x}$ ). For a subset $X \subset \mathcal{P}$ and a matrix corepresentation $M$, set $M_{X}=\bigcup_{x \in X} M_{x}$.

For any $\mathbb{F}$-subspace $U$ of some linear $\mathbb{G}$-space $V$, we denote by $\widetilde{U}$ and $\underset{\sim}{U}$ respectively the $\mathbb{G}$-hull and $\mathbb{G}$-cohull of $U$ defined as follows:

$$
\tilde{U}=\bigcap_{U \subset W} W, \quad \quad \underset{\sim}{ }=\sum_{W \subset U} W,
$$

where $W$ are $\mathbb{G}$-subspaces of $V$. It is clear that $\tilde{U}$ (resp. $\underset{\sim}{U}$ ) is the minimal (maximal) $\mathbb{G}$-subspace of $V$ containing $U$ (contained in $U$ ). Moreover, one can consider $\widetilde{U}$ as the ordinary $\mathbb{G}$-span of $U$ i.e., $\widetilde{U}=\mathbb{G} U$. When $U$ itself is a $\mathbb{G}$-subspace then $\tilde{U}=\underset{\sim}{U}=U$ and $U$ is said to be $\mathbb{G}$-strong or strong.

A corepresentation of an equipped poset $\mathcal{P}$ over the pair $(\mathbb{F}, \mathbb{G})$ is a collection of the form $U=\left(U_{0}, U_{x}: x \in \mathcal{P}\right)$ where $U_{0}$ is a finitedimensional $\mathbb{G}$-space containing $\mathbb{F}$-subspaces $U_{x}$ such that

$$
\begin{aligned}
& x \leq y \Longrightarrow U_{x} \subset U_{y}, \\
& x \triangleleft y \Longrightarrow \widetilde{U}_{x} \subset U_{y} .
\end{aligned}
$$

Notice that if $x \in \mathcal{P}^{\circ}$ then $U_{x}$ is a strong subspace.

The dimension of $U$ is a vector $d=\underline{\operatorname{dim}} U=\left(d_{0}, d_{x}: x \in \mathcal{P}\right)$ with $d_{0}=\operatorname{dim}_{\mathbb{G}} U_{0}$ and $\left.d_{x}=\operatorname{dim}_{\mathbb{G}} U_{x} / \underline{U_{x}} \overline{(\operatorname{resp} .} d_{x}=\operatorname{dim}_{\mathbb{F}} U_{x} / \underline{U_{x}}\right)$ for a strong (weak) point $x$, where

$$
\underline{U_{x}}=\sum_{\substack{y \prec x \\ y \neq x}} U_{y}+\sum_{\substack{y \triangleleft x \\ y \neq x}} \tilde{U}_{y}
$$

is the radical subspace of $U_{x}$.

Corepresentations are the objects of the category $\operatorname{corep}_{(\mathbb{F}, \mathbb{G})}(\mathcal{P}, \leq$ $, \triangleleft)$, briefly corep $\mathcal{P}$, with morphisms $U \stackrel{\varphi}{\rightarrow} V$ being $\mathbb{G}$-linear maps $\varphi: U_{0} \rightarrow V_{0}$ such that $\varphi\left(U_{x}\right) \subset V_{x}$ for each $x \in \mathcal{P}$. It is clear that 
two corepresentations $U, V$ are isomorphic if and only if for some $\mathbb{G}$ isomorphism $\varphi: U_{0} \rightarrow V_{0}$ it holds $\varphi\left(U_{x}\right)=V_{x}$ for all $x$.

The classification of indecomposable objects of the category corep $\mathcal{P}$, up to isomorphism, corresponds precisely to the described above matrix problem $(a)-(c)$.

Namely, if $M$ is a matrix corepresentation of $\mathcal{P}$ with $\operatorname{dim} M=$ $\left(d_{0}, d_{x}: x \in \mathcal{P}\right)$, one may consider a base $e_{1}, \ldots, e_{d_{0}}$ of some $d_{0^{-}}$ dimensional $\mathbb{G}$-space $U_{0}$ and identify each column $\left(\lambda_{1}, \ldots, \lambda_{d_{0}}\right)^{T}$ of $M$ with the element $u=\lambda_{1} e_{1}+\cdots+\lambda_{d_{0}} e_{d_{0}} \in U_{0}$. Given any column set $X \subset M$, denote by $\mathbb{F}[X]$ and $\mathbb{G}[X]$ the $\mathbb{F}$-span and $\mathbb{G}$-span of $X$ in $U_{0}$ respectively. Then, one can form a corepresentation $U_{M}=$ $\left(U_{0}, U_{x}: x \in \mathcal{P}\right)$ where $U_{x}=\sum_{y \prec x} \mathbb{F}\left[M_{y}\right]+\sum_{y \triangleleft x} \mathbb{G}\left[M_{y}\right]$, which satisfies the conditions (2.3).

From this point of view, the columns of each vertical stripe $M_{x}$ represent a system of generators of the space $U_{x}$ modulo its radical subspace

$$
\underline{U_{x}}=\sum_{\substack{y \prec x \\ y \neq x}} \mathbb{F}\left[M_{y}\right]+\sum_{\substack{y \triangleleft x \\ y \neq x}} \mathbb{G}\left[M_{y}\right] .
$$

Hence, the transformations $(a)-(c)$ of $M$ reflect both base changing in $U_{0}$ and generator changing in subspaces $U_{x}$. Conversely, starting from $U$, one can associate with $U$ a matrix corepresentation denoted by $M_{U}$. Notice that $\underline{\operatorname{dim}} U_{M} \leq \underline{\operatorname{dim}} M$ and $\underline{\operatorname{dim}} U \leq \underline{\operatorname{dim}} M_{U}$, in both cases the equality holds if and only if the columns of each stripe $M_{x}$ are linearly independent modulo the radical column. When dealing with matrix corepresentations $M_{U}$ corresponding to corepresentations $U$, we always will select such matrices $M=M_{U}$ that $\underline{\operatorname{dim}} M=\underline{\operatorname{dim}} U$.

Given any corepresentation $U$ of $\mathcal{P}$ with dimension $d=\operatorname{dim} U$, the support of $U$ is the subset $\operatorname{Supp} U=\left\{x \in \mathcal{P}: d_{x}>0\right\}$. $U$ will be called trivial if $\operatorname{dim}_{\mathbb{G}} U_{0}=1$.

A vector $d$ is sincere if has no zero coordinates. We call a corepresentation sincere if its dimension vector is sincere. Every equipped poset having at least one sincere indecomposable corepresentation is called sincere (with respect to corepresentations).

There exists an analogous definition of a matrix corepresentation and of a corepresentation of a poset $\mathcal{P}$ over the pair $(\mathbb{F}[t], \mathbb{G}[t])$ of polinomial rings. 
An $(\mathbb{F}[t], \mathbb{G}[t])$-corepresentation of $\mathcal{P}$ is a collection $U(t)=\left(U_{0}, U_{x}\right.$ : $x \in \mathcal{P})$ such that $U_{0}$ is a finitely generated free $\mathbb{G}[t]$-module and each of $U_{x}$ is a finitely generated $\mathbb{F}[t]$-submodule of $U_{0}$, in particular the $\mathbb{G}[t]$-hull of any finitely generated $\mathbb{F}$-submodule $V$ is the $\mathbb{G}[t]$-span of $V$.

An $(\mathbb{F}[t], \mathbb{G}[t])$-matrix corepresentation of $\mathcal{P}$ is a rectangular matrix $M[t]$ over $\mathbb{G}[t]$ separated into vertical stripes $M_{x}[t](x \in \mathcal{P})$, the admissible transformations are analogously defined to $(a)-(c)$, but over the pair $(\mathbb{F}[t], \mathbb{G}[t])$.

A corepresentation series is obtained from a matrix $M[t]$ of $(\mathbb{F}[t], \mathbb{G}[t])$-corepresentation by substituting any square matrix $A$ over $\mathbb{G}$ for the variable $t$ and scalar matrices $\lambda I$ of the same size for the coefficients $\lambda \in \mathbb{G}$. A corepresentation series of $\mathcal{P}$ is called sincere if it induces at least a sincere indecomposable corepresentation of $\mathcal{P}$.

A poset of infinite type is said to be one-parametric if it has a series containing almost all indecomposable corepresentations of each given dimension.

Denote by $\mathcal{P} o p$ the antiisomorphic poset to a poset $\mathcal{P}$. Given a corepresentation $U$ of $\mathcal{P}$, we define the dual corepresentation $U^{*}$ of $\mathcal{P}^{o p}$ by setting

$$
U^{*}=\left(U_{0}^{*}, U_{x}^{*}: x \in \mathcal{P}^{o p}\right),
$$

where $U_{0}^{*}=\operatorname{Hom}_{\mathbb{G}}\left(U_{0}, \mathbb{G}\right)$ and $U_{x}^{*}= \begin{cases}\left(U_{x}\right)_{\mathbb{G}}^{\perp}=\left\{\varphi \in \operatorname{Hom}_{\mathbb{G}}\left(U_{0}, \mathbb{G}\right): U_{x} \subset \operatorname{Ker} \varphi\right\}, & \text { if } x \text { is strong; } \\ \left(U_{x}\right)_{\mathbb{F}}^{\perp}=\left\{\varphi \in \operatorname{Hom}_{\mathbb{F}}\left(U_{0}, \mathbb{F}\right): U_{x} \subset \operatorname{Ker} \varphi\right\}, & \text { if } x \text { is weak. }\end{cases}$

It can be established easily that an equipped poset $\mathcal{P}$ is sincere if and only if $\mathcal{P}^{o p}$ is.

\section{Formulation of the Main Results}

The main goal of the present investigation is to obtain a complete classification of corepresentations of some one parametric equipped posets ( $K_{6}, K_{8}, A_{25}, A_{28}, A_{38}, A_{41}$ in the notation of [24]), as well as to establish several properties of corepresentations of equipped posets of infinite type (not necessarily of one parameter type) containing a well inserted critical subset $K_{6}$ or $K_{8}$ of the form of Figure 2. 


$\begin{array}{cccccc}\otimes & \otimes & 0 & 0 & \otimes \\ a & b & r & s & a \\ & & & & & \\ & K_{6} & & & \end{array}$

Figure 2. Critical posets $K_{6}$ and $K_{8}$.

In order to do this, we use in particular, the algorithm of differentiation $\widehat{\mathrm{VII}}$ developed in [20], as well as the classical algorithm of differentiation of ordinary posets with respect to a maximal point [14] and some special methods of reduction of matrix problems of mixed type over the pair $(\mathbb{F}, \mathbb{G})$. Naturally, we develop also some combinatorial technique concerning equipped posets and their quadratic forms. Now, we present the formulations of the main results of the paper which will be proved in Sections 4 and 5 .

3.1. Properties of well inserted subposet of type $K_{6}$. A subposet $K_{6} \subset \mathcal{P}$, as in Figure 2, is said to be well inserted in $\mathcal{P}$ if the subsets

$(a \curlyvee b)=\{x \in \mathcal{P}: a \supsetneqq x, b \supsetneqq x\}, \quad(a \curlywedge b)=\{x \in \mathcal{P}: x \supsetneqq a, x \supsetneqq b\}$ are chains and each of the subsets $N(a)$ and $N(b)$ (of type (2.2)) is a chain with a unique weak point, see Figure 3(a) below in which $c_{1}, \ldots, c_{4}$ are ordinary chains and $X, Y$ are completely weak chains.
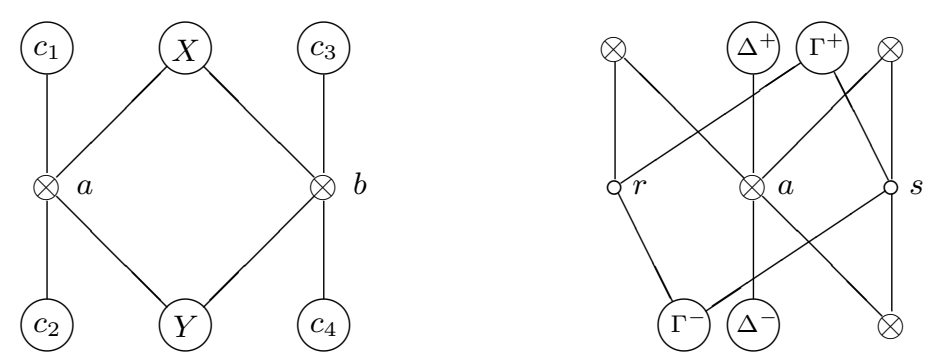

FiguRE 3. (a) Example of a well inserted subposet of type $K_{6}$. (b) Example of a well inserted subposet of type $K_{8}$.

Theorem 3.1. If an equipped poset $\mathcal{P}$ contains a well inserted subposet $K_{6}$, then each indecomposable (not necessarily sincere) corepresentation $U$ of $\mathcal{P}$ satisfies the following conditions: 


$$
\begin{aligned}
& \text { (1) } U_{a} \cap U_{b}=0 \quad \text { or } \quad \tilde{U}_{a}=\tilde{U}_{b}=U_{0} \text {; } \\
& \text { (2) } U_{a}+U_{b}=U_{0} \quad \text { or } \quad U_{a}=U_{b}=0 .
\end{aligned}
$$

Corollary 3.2. Assume that an equipped poset $\mathcal{P}$ contains a well inserted subposet $K_{6}$ as above and two points $p, q$ such that $q \triangleleft a$ and $\{a, b\}<p$, or $q<\{a, b\}$ and $a \triangleleft p$. Then each indecomposable corepresentation of $\mathcal{P}$ is not sincere at one of the points $p, q$.

3.2. Properties of well inserted subposet of type $K_{8}$. A poset $\mathcal{P}$ has a well inserted subposet $K_{8}$ as in Figure 2, if the following conditions are satisfied (see an example in Figure 3(b)):

(1) $N(a)=\left\{\Gamma^{-}<\{r, s\}<\Gamma^{+}\right\}$where $\Gamma^{-}, \Gamma^{+}$are ordinary chains.

(2) $N(r, s)=\left\{\Delta^{-}<a<\Delta^{+}\right\}$where $\Delta^{-}, \Delta^{+}$are ordinary chains.

(3) The sets

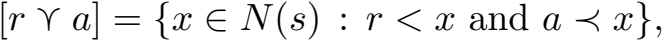

$$
\begin{aligned}
& {[r \curlywedge a]=\{x \in N(s): x<r \text { and } x \prec a\}}
\end{aligned}
$$

and the defined analogously sets $[s \curlyvee a]$ and $[s \curlywedge a]$ are chains, such that $\left|\left[\begin{array}{lrl}r \curlyvee a & \gamma\end{array}\right]\left[\begin{array}{lll}s & \curlyvee & a\end{array}\right]\right| \leq 2$ and $\left|\left[\begin{array}{rll}r \curlywedge & a\end{array}\right]+\left[\begin{array}{lll}s \curlywedge & a\end{array}\right]\right| \leq 2$.

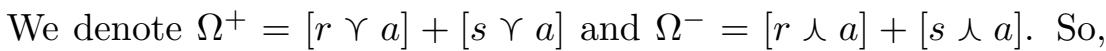
one can suppose that a poset $\mathcal{P}$ with a well inserted subposet $K_{8}$ has the form

$$
\mathcal{P}=\Omega^{+}+\Omega^{-}+\Gamma^{+}+\Gamma^{-}+\Delta^{+}+\Delta^{-}+X,
$$

for certain subset $X$.

Theorem 3.3. If an equipped poset $\mathcal{P}$ contains a well inserted subposet $K_{8}$, then each indecomposable (not necessarily sincere) corepresentation $U$ of $\mathcal{P}$ satisfies the following conditions:

$$
\begin{aligned}
& \text { (1) } U_{a} \cap U_{r}=U_{a} \cap U_{s}=0 \quad \text { or } \quad \widetilde{U}_{a}=U_{r}+U_{s}=U_{0} \text {; } \\
& \text { (2) } U_{a}+U_{r}=U_{a}+U_{s}=U_{0} \quad \text { or } \quad U_{a}=U_{r} \cap U_{s}=0 .
\end{aligned}
$$

Corollary 3.4. Let an equipped poset $\mathcal{P}$ contains a well inserted subposet $K_{8}$ as above and points $p, q$ satisfying one of two conditions:

$$
\begin{aligned}
& \text { (1) } q \triangleleft a \text { or } q<\{r, s\}, \text { and }\{a, r\}<p \text { or }\{a, s\}<p \text {; } \\
& \text { (2) } q<\{a, r\} \text { or } q<\{a, s\}, \text { and } a \triangleleft p \text { or }\{r, s\}<p \text {. }
\end{aligned}
$$

Then each indecomposable corepresentation of the poset $\mathcal{P}$ is not sincere at one of the points $p, q$. 
3.3. Classification of indecomposable corepresentations of some critical equipped posets. We present the complete matrix classification of indecomposable corepresentations of the critical posets $K_{6}$ and $K_{8}$ for an arbitrary quadratic field extension $\mathbb{F} \subset \mathbb{G}$.

In particular, the calculation of the corepresentation series of $K_{6}$ is reduced to the classical matrix pencil problem, solved completely by Kronecker [13] (see [9], [26] for recent short solutions).

On the other hand, the corepresentation series of $K_{8}$ is reduced to an homogeneous biquadratic matrix problem which is closely related to the semilinear and pseudolinear matrix pencil problems, observed for instance in [26], [27].

Also we establish a bijective correspondence between certain indecomposable corepresentations and the positive roots of the Tits quadratic form.

Theorem 3.5. The critical poset $K_{6}$ possesses 6 types of indecomposable corepresentations over the pair $(\mathbb{F}, \mathbb{G}$ ) (up to duality and permutation of points), listed together with their duals in the matrix form in Appendix A.

Theorem 3.6. The critical poset $K_{8}$ possesses 11 types of indecomposable corepresentations over an arbitrary quadratic field extension $\mathbb{F} \subset \mathbb{G}$ (up to duality and permutation of strong points $r, s$ ), listed together with their duals in Appendix B.

Given an equipped poset $\mathcal{P}$, we set $\mathcal{P}^{\bullet}=\mathcal{P} \cup\{0\} \quad(0$ is a formal symbol incomparable with the points in $\mathcal{P})$. A vector $e_{x} \in \mathbb{Z}^{\mathfrak{P} \bullet}(x \in$ $\left.\mathcal{P}^{\bullet}\right)$ is said to be a simple root if $\left(e_{x}\right)_{x}=1$ and $\left(e_{x}\right)_{y}=0$ for $x \neq y$. The Tits quadratic form $f_{\mathcal{P}}=f$ associated to $\mathcal{P}$ is defined on a given vector $d=\left(d_{0}, d_{x}: x \in \mathcal{P}\right)$ by the formula

$$
f(d)=\sum_{x, y \in \mathcal{P}^{0}}\left(l_{x y} d_{x} d_{y}\right)-2 d_{0} \sum_{x \in \mathcal{P}} d_{x}
$$

where $l_{x y}=0$ if $x \gtrless y, \quad l_{x y}=1$ if $x \prec y, \quad$ and $l_{x y}=2$ if $x \triangleleft y$ or $x=y=0$.

Let $\langle x, y\rangle=\frac{1}{4}[f(x+y)-f(x-y)]$ be the corresponding symmetric bilinear form. We recall that the reflection at the point $x \in \mathcal{P}^{\bullet}$ is a mapping $\rho_{x}: \mathbb{Z}^{\mathfrak{P} \bullet} \longmapsto \mathbb{Z}^{\mathfrak{P} \bullet}$ defined by 


$$
\rho_{x}(d)=d-\frac{2}{l_{x x}}\left\langle d, e_{x}\right\rangle \cdot e_{x} .
$$

The roots of the Tits form $f_{\mathcal{P}}$ (of the poset $\mathcal{P}$ ) are the vectors obtained by reflections from simple roots. Let $d, d^{\prime}$ be roots of $f_{\mathcal{P}}$, we write $d \leq d^{\prime}$ if $d_{x} \leq d_{x}^{\prime}\left(x \in \mathcal{P}^{\bullet}\right)$. We will deal mainly with admissible roots which are positive roots with $d_{0}>0$.

A vector $\mu \geq \mathbf{0}$ is said to be an imaginary root of $f_{\mathcal{P}}$ or of $\mathcal{P}$ if $f_{\mathcal{P}}(\mu)=0$. Two roots $d, d^{\prime}$ of $f_{\mathcal{P}}$ are said to be of the same type if $d=d^{\prime}+\mu$, where $\mu$ is an imaginary root. We refer to $\mu_{0}$ as The minimal root in the set of roots of the same type.

We will consider now the following sincere equipped posets
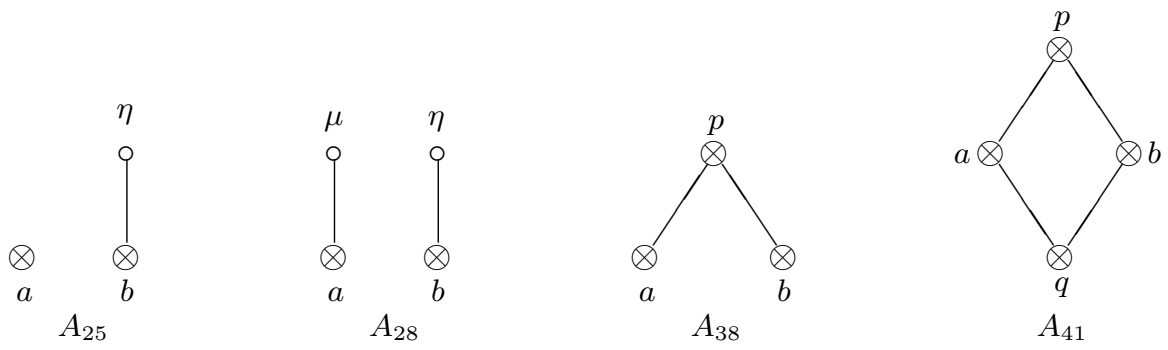

FiguRE 4. Some sincere one parametric equipped posets.

Theorem 3.7. Let $\mathcal{P}$ be one of the posets $K_{6}, K_{8}, A_{25}, A_{28}, A_{38}, A_{41}$ or their duals shown in Figure 2 and 4. Then there exists a bijection between the admissible roots of $f_{\mathcal{P}}$ and the isoclasses of indecomposable corepresentations of $\mathcal{P}$ whose dimension is not an imaginary root.

Corollary 3.8. The value of the Tits form on the dimension of any indecomposable corepresentation of any of the posets $K_{6}, K_{8}, A_{25}$, $A_{28}, A_{38}, A_{41}$ or their duals is 0,1 or 2 .

the minimal admissible roots of the Tits form of the mentioned posets in Theorem 3.7 are listed in Appendix E. 


\section{Classification of Corepresentations of some One Para- metric Equipped Posets}

We present a complete classification (in matrix form) of the indecomposable corepresentations of the sincere equipped posets $K_{6}, K_{8}$, $A_{25}, A_{28}, A_{38}, A_{41}$ and their duals over an arbitrary quadratic field extension $\mathbb{F} \subset \mathbb{F}(\xi)=\mathbb{G}$. Doing this, we distinguish separable and inseparable extensions, as well as take into account the characteristic of the fields.

To obtain this classification, we use the known functors of differentiation of ordinary posets, D-I, D- $\widehat{\mathrm{VII}}$ constructed in [25] and [20], as well as some special reduction methods for mixed matrix problems over the considered pair of fields $(\mathbb{F}, \mathbb{G})$.

Remark that the corepresentation series of $K_{8}$ is expressed in terms of the solution either of the semilinear in [5] or of the pseudolinear (originally [21]) pencil problem. These problems were considered recently with details in [26] and [27].

4.1. The case of posets $K_{6}, A_{25}, A_{28}$, and of their duals. The classification of indecomposable corepresentations of $K_{6}$ is obtained by considering three cases.

Proof of Theorem 3.5. Let $U$ be an indecomposable corepresentation of $K_{6}$.

First case: $\widetilde{U}_{b} \neq U_{0}$. Let $V$ be the indecomposable corepresentation of $A_{25}$ defined by $V_{0}=U_{0}, V_{x}=U_{x}(x \in\{a, b\})$ and $V_{\eta}=\widetilde{U}_{b}$. Since $\operatorname{dim} V_{\eta}<\operatorname{dim} V_{0}$, if we apply to the poset $A_{25}$ the $\widehat{\mathrm{VII}}-$ differentiation with respect to the pair $(a, \eta)$ then $\operatorname{dim}_{\mathbb{G}} V_{0}^{\prime}<\operatorname{dim}_{\mathbb{G}} V_{0}$ (the dimension of the principal space of the derived corepresentation of $V)$.

Additionally, Supp $V^{\prime} \subset\left\{a^{-} \triangleleft a^{+}, b\right\}$ (see Figure 5), thus we can manage with the induction step. Therefore, we must $\widehat{\text { VII-integrate }}$ the indecomposable corepresentations of $A_{25}^{\prime}$ (not necessarily sincere) exhaustively, obtaining a complete classification of indecomposable corepresentations of $A_{25}$ and the indecomposable corepresentations of $K_{6}$ satisfying the above condition. 


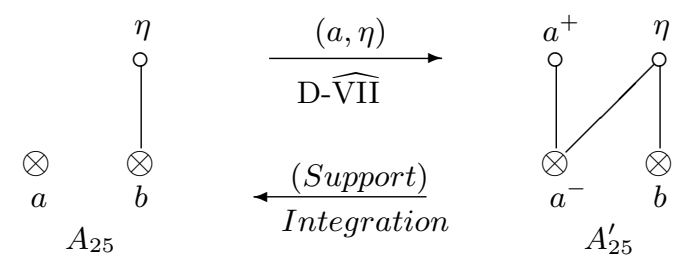

Figure 5. Combinatorial $\widehat{V I I}-$ differentiation of the poset $A_{25}$.

The finite poset $F_{14}=\{\mathrm{o}, \otimes\}$ is a subposet of $A_{25}^{\prime}$ (by considering the points $a^{+}$and $b$ ), then any corepresentation of $F_{14}$ can be expanded of natural form to some corepresentation of $A_{25}^{\prime}$.

We number the types of indecomposable corepresentation and denote the $i$-th type by $\mathcal{P}-i$.

We stated that the indecomposable corepresentations ${ }^{1} F_{14}-1, F_{14}-2$ and $F_{14}-3$ induce the indecomposable corepresentations $K_{6}-1^{*}, K_{6}$ $2^{*}, K_{6}-3, K_{6}-4$, and $A_{25}-1, \ldots, A_{25}-5$. Moreover there do not exist another with the same property.

The integration process is shown in Figure 6. For every arrow, we integrate a corepresentation of $A_{25}^{\prime}$ (the source), and obtain a corepresentation of $A_{25}$ (the target), the support is over the arrow. Source and target (denoted by capital letters) are extended corepresentations from the corepresentations placed below. Also, we present their dimensions.

Second case: $\widetilde{U}_{b} \neq U_{0}$. Then $U$ is analogously extended to some corepresentation of $A_{25}^{*}$ and by dual considerations we obtain the classification of indecomposable corepresentations of $A_{25}^{\prime}$ and some indecomposable corepresentations types of $K_{6}$ satisfying the above condition.

Third case: $\widetilde{U}_{b}=U_{0}$ and ${\underset{\sim}{b}}_{b}=0$. Therefore, the associated ma-

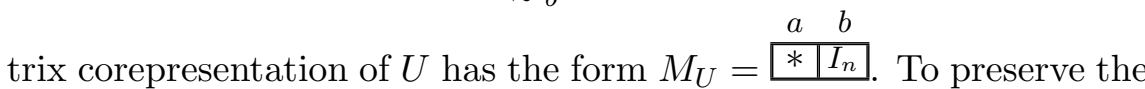
identity block it is allowed to apply $\mathbb{F}$-elementary row transformations

\footnotetext{
${ }^{1}$ This is a finite poset having three sincere indecomposable corepresentations,

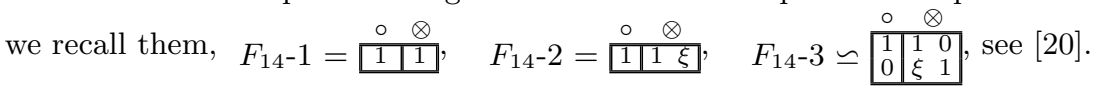




$$
\begin{aligned}
& \begin{array}{cccccc}
\text { Int } & A \stackrel{\left(a^{+}, b\right)}{\longrightarrow} & A_{0} \stackrel{\left(a^{-}, b\right)}{\longrightarrow} & A_{1} \stackrel{\left(b, a^{-}, a^{+}\right)}{\longrightarrow} & A_{2} \stackrel{\left(a^{-}, b\right)}{\longrightarrow} & A_{3} \stackrel{\left(b, a^{-}, a^{+}\right)}{\longrightarrow} \cdots \\
\text { corep } & F_{14^{-1}} & K_{6^{-1}} & A_{25^{-1}} & K_{6^{-1}} &
\end{array} \\
& \operatorname{dim}(1,1,1) \quad(2,2,1) \quad(2,2,1,1) \quad(3,3,2) \quad(3,3,2,1)
\end{aligned}
$$

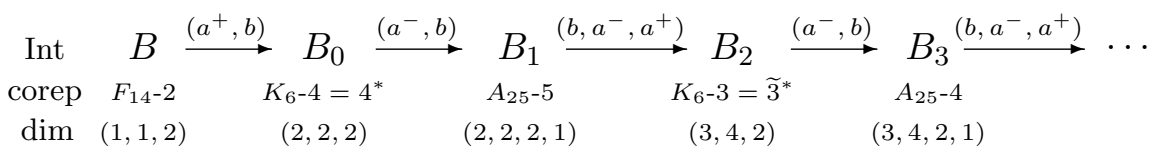

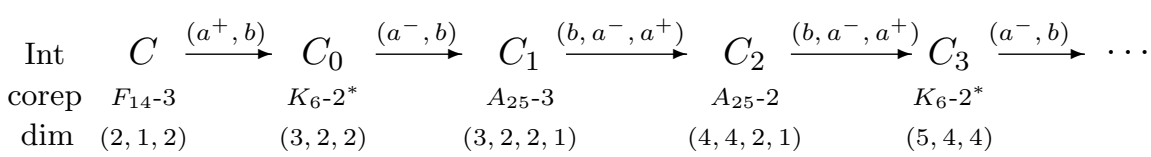

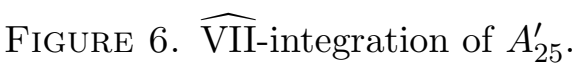

over whole matrix and independent $\mathbb{F}$-elementary column transformations over the blocks $M_{a}$ and $M_{b}$.

This problem precisely corresponds to the classical pencil problem of Kronecker over the field $\mathbb{F}$, namely, since the square block $M_{a}$ has coefficients over $\mathbb{G}$, we set $A=\operatorname{Re}\left(M_{a}\right)$ and $B=\operatorname{Im}\left(M_{a}\right)$, they are matrices over $\mathbb{F}$ and the elementary transformations above correspond to the following simultaneous similarity transformation

$$
(A, B) \longmapsto\left(X^{-1} A Y, X^{-1} B Y\right)
$$

where $X, Y$ are non singular square matrices over $\mathbb{F}$.

The four types of solutions induce the indecomposable corepresentations of $K_{6}$ of types $\widetilde{1}, \widetilde{1}^{*}, 5$, and 6 (the series).

The corepresentation $K_{6}-\widetilde{i}$ (resp. $K_{8}-\widetilde{j}$ ) is obtained from $K_{6}-i$ $\left(K_{8}-j\right)$ by switching the point $a$ with $b(r$ with $s)$ and viceversa.

The classification of corepresentations of $A_{25}$ follows from the above considerations, the classification of corepresentations of $A_{25}^{*}$ is obtained directly by applying duality, therefore it holds the following result.

Proposition 4.1. Each of the posets $A_{25}$ and $A_{25}^{*}$ has 5 types of indecomposable corepresentations sincere at the point $\eta$ listed in Appendix C. 


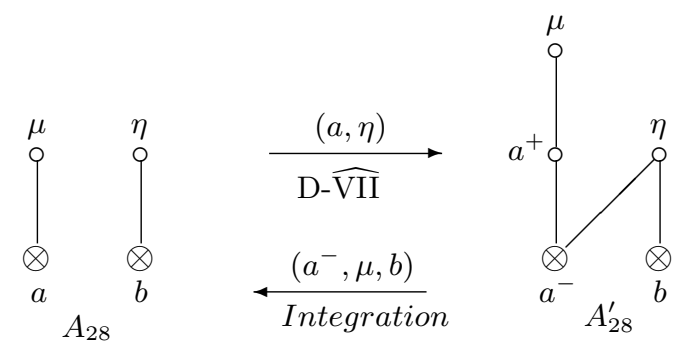

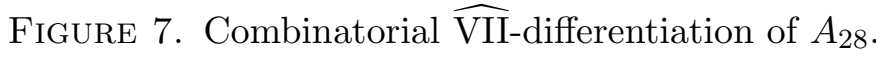

The classifications of indecomposable corepresentations of $A_{28}$ and $A_{28}^{*}$ are obtained analogously to the case of $A_{25}$ and $A_{25}^{*}$ respectively. The poset $A_{28}$ has a $\widehat{\mathrm{VII}}$-suitable pair $(a, \eta)$, then we manage by induction on dimension. In particular, its unique indecomposable corepresentation is obtained from $A_{25}-3$, considered as a corepresentation of $A_{28}^{\prime}$ with support $\left\{a^{-}, \mu, b\right\}$, see Figure 7 .

Proposition 4.2. Each of the posets $A_{28}$ and $A_{28}^{*}$ has 1 type, up to automorphism of equipped posets, of indecomposable corepresentations sincere at the points $\mu, \eta$ having the following matrix forms in the notations of Section 6.

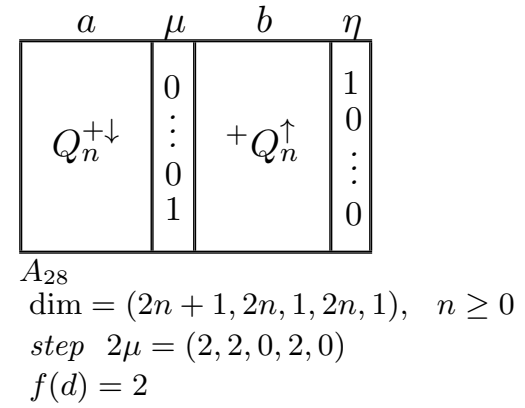

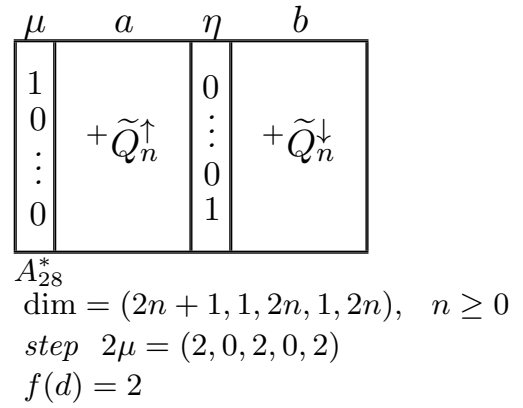

4.2. The case of posets $A_{38}, A_{38}^{*}$, and $A_{41}$. We reduce the matrix problem on classification of indecomposable corepresentations of $A_{38}$, $A_{38}^{*}$, and $A_{41}$ to the problem on classification of indecomposable representations, in the sense of [22], of the poset $K_{8}$. Additionally we confirm the classification of corepresentations of $K_{6}$. 
Let $M$ be an indecomposable matrix representation ${ }^{2}$ of $K_{8}$. Since $\{r, s\} \subset K_{8}$ is an ordinary subposet, the blocks $M_{r}, M_{s}$ can be reduced as an ordinary dyad. Notice that $M_{a}$ must be indecomposable and it has coefficients over $\mathbb{G}$.

We assign to every horizontal stripe of $M$ one point of the poset $A_{41}$ as in Figure 8. To preserve the reduced blocks $M_{r}$ and $M_{s}$ we can apply $\mathbb{G}$-elementary column transformations to the block $M_{a}$, independent $\mathbb{F}$-elementary row transformations of each horizontal stripe and row additions over $\mathbb{F}$ between the horizontal stripes which are suggested by the poset $A_{41}$.

Therefore, we establish a bijective correspondence between indecomposable representations of $K_{8}$ and indecomposable corepresentations of the subposets of $A_{41}$.

Remark 3. If $M$ is an indecomposable matrix corepresentation of $K_{8}$, we may reduce it as in the representation case. Then we establish an analogous relationship between indecomposable corepresentations of $K_{8}$ and indecomposable representations of $A_{38}, A_{38}^{*}, A_{41}$ and $K_{6}$.

The Figure 8 shows the matrix $M$ both for the representation and for the corepresentation case. The fields within parentheses correspond to representation case, the outside fields correspond to the corepresentation case.

\footnotetext{
${ }^{2}$ The blocks $M_{r}, M_{s}$ have coefficients over $\mathbb{F}$, and $M_{a}$ over $\mathbb{G}$, additionally we can apply $\mathbb{F}$-elementary row transformations of whole matrix as well as independent $\mathbb{F}$-elementary ( $\mathbb{G}$-elementary) column transformations to the stripe corresponding to the points $r, s$ (resp. to the point $a$ ).
} 


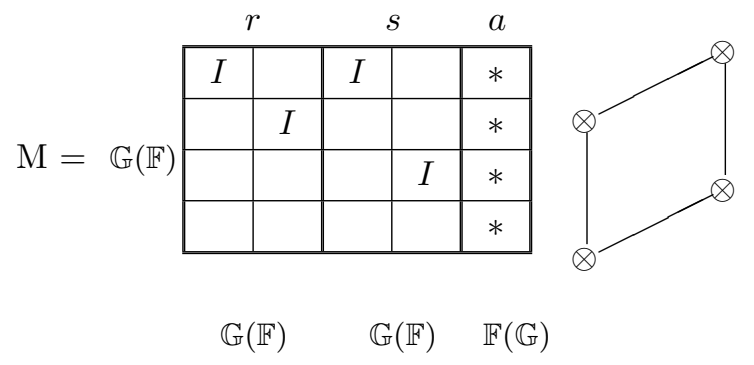

FiguRE 8. Indecomposable reduced matrix corepresentation (representation) $M$ of $K_{8}$ (the empty blocks denote zero blocks).

Remark 4. Notice that a sincere non trivial indecomposable representation of $K_{8}$ just corresponds to some sincere indecomposable corepresentation of $A_{38}, A_{38}^{*}, A_{41}$ or $K_{6}$. Furthermore, the representation series of $K_{8}$ is related to the corepresentation series of $K_{6}$, then they are reduced to the classical pencil problem over $\mathbb{F}$.

\begin{tabular}{|c|c|}
\hline Condition & $\begin{array}{l}U \text { induces indecomposable representa- } \\
\text { tions (corepresentations) of }\end{array}$ \\
\hline$U_{r} \cap U_{s}=0$ and $U_{r}+U_{s}=U_{0}$ & $K_{6}$ \\
$U_{r} \cap U_{s} \neq 0$ and $U_{r}+U_{s}=U_{0}$ & $A_{38}$ \\
$U_{r} \cap U_{s}=0$ and $U_{r}+U_{s} \neq U_{0}$ & $A_{38}^{*}$ \\
$U_{r} \cap U_{s} \neq 0$ and $U_{r}+U_{s} \neq U_{0}$ & $A_{41}$ \\
\hline
\end{tabular}

TABLE 1. Relationship between posets $K_{8}$ and $A_{38}$, $A_{38}^{*}, A_{41}$, and $K_{6}$.

We reformulate in Table 1 the described above relationship in the invariant language. Where $U$ is an indecomposable corepresentation (resp. representation) of $K_{8}$.

Our computations are based on the classification of representations of $K_{8}$ over the pair $(\mathbb{R}, \mathbb{C})$ given in [22]. This classification was obtained by using some differentiation techniques valid in fact for any quadratic field extension $(\mathbb{F}, \mathbb{G})$. So, the matrix forms of indecomposable representations described in $[22]$ over the pair $(\mathbb{R}, \mathbb{C})$ coincide 
with those over the pair $(\mathbb{F}, \mathbb{G})$. Therefore, we state the following results.

Proposition 4.3. Each of the posets $A_{38}$ and $A_{38}^{*}$ has 4 types of indecomposable corepresentations sincere at the points $p$ and $q$ respectively (up to automorphisms of equipped posets). They are listed in appendix D.

Proposition 4.4. The poset $A_{41}=\{p \prec\{a, b\} \prec q\}$ has only 1 type of indecomposable corepresentation, with the following matrix form

\begin{tabular}{|c|c|c|c|}
\multicolumn{1}{c}{$q$} & $a$ & $b$ & $p$ \\
\hline 1 & & & 0 \\
0 & & & $I_{n}^{\uparrow}$ \\
$\vdots$ & $I_{n}^{\uparrow}+\xi I_{n}^{\downarrow}$ & $\vdots$ \\
0 & & & $\xi$ \\
\hline
\end{tabular}

$$
\begin{aligned}
& \operatorname{dim}=(n+1,1, n, n, 1), \quad n \geq 0 \\
& \text { step } \quad \mu=(1,0,1,1,0) \\
& f(d)=1
\end{aligned}
$$

4.3. The case of $K_{8}$. All indecomposable corepresentation types of $K_{8}$ are induced directly by indecomposable representations types of $A_{38}, A_{38}^{*}, A_{41}$, and $K_{6}$.

Although the classification of representations of them was made over the pair $(\mathbb{R}, \mathbb{C})$, see [22], many indecomposable matrix types coincide with the indecomposable ones over the pair $(\mathbb{F}, \mathbb{G})$ by setting $\xi=\mathbf{i}$. The classification of this representations were obtained by using differentiation techniques which are valid for an arbitrary quadratic field extension.

However, the representation series of $K_{6}$ over the pair $(\mathbb{F}, \mathbb{G})$ is reduced to some biquadratic matrix problem over $\mathbb{F}$, in the sense of $[26],[27]$.

Proof of Theorem 3.6. Let $\mathbb{F} \subset \mathbb{F}(\xi)=\mathbb{G}$ be an arbitrary quadratic field extension with generator $\xi$ and minimal polynomial $\wp(t)=t^{2}+$ $p t+q$. The conjugate roots of $\wp(t)$ are $\xi$ and $\bar{\xi}=-\xi-p$, in general 
$\overline{a+\xi b}=a-p b-\xi b(a, b \in \mathbb{F})$.

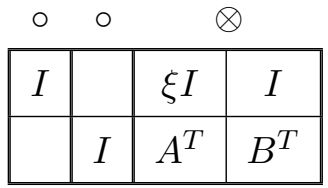

(a)

\begin{tabular}{|c|c|}
\hline \multirow{3}{*}{$\otimes$} & $\otimes$ \\
\hline$I$ & $B$ \\
\hline \hline
\end{tabular}

(b)

\begin{tabular}{|l|l|}
\hline$A_{1}$ & $A_{2}$ \\
\hline$B_{1}$ & $B_{2}$ \\
\hline
\end{tabular}

(c)

Since the representation series of $K_{6}$ is obtained in [22] by considering a matrix $M$ of the form (4.1)(b), the corepresentation series of $K_{8}$ has the form (4.1)(a).

Notice that the blocks $A$ and $B$ have coefficients over $\mathbb{G}$. So, the problem consists in finding a canonical matrix form for the blocks $A$ and $B$ by using admissible transformations that preserve the reduced block $\frac{\xi I}{I}$

$$
\begin{array}{|l|}
\hline \xi I \\
\hline I \\
\hline
\end{array}
$$

The above problem is equivalent to the following matrix problem over $\mathbb{F}$. Consider a matrix of the form (4.1)(c) where $\operatorname{Im}(A)=A_{1}$, $\operatorname{Re}(A)=A_{2}, \operatorname{Im}(B)=B_{1}$ and $\operatorname{Re}(B)=B_{2}$, then the admissible transformations over a block of the form (4.1) (c) are given by the solutions of the equations (4.2) and (4.3) for the blocks $X_{1}, \ldots, X_{4}$ and $Y_{1}, \ldots, Y_{4}$

$$
\begin{aligned}
& \begin{array}{|l|l|}
\hline X_{1} & X_{2} \\
\hline X_{3} & X_{4} \\
\hline
\end{array} \cdot \begin{array}{|c|}
\xi \xi I \\
\hline I
\end{array}=\begin{array}{|c|}
\xi I \\
\hline I
\end{array} \cdot \overline{U+\xi V}, \\
& \begin{array}{|l|l|}
\hline A_{1} & A_{2} \\
\hline B_{1} & B_{2} \\
\hline
\end{array} \cdot \begin{array}{|l|l|}
\hline Y_{1} & Y_{2} \\
\hline Y_{3} & Y_{4} \\
\hline
\end{array}=\begin{array}{|l|}
\xi A_{1}+A_{2} \\
\hline \xi B_{1}+B_{2} \\
\hline Z+\xi T, \\
Z
\end{array}
\end{aligned}
$$

where $\overline{U+\xi V}$ and $Z+\xi T$ are square $\mathbb{G}$-matrices. The equivalent problem consists in finding a canonical form for block matrices of type (4.1)(c) with respect to the following transformation

$$
\begin{array}{|c|c|}
\hline A_{1} & A_{2} \\
\hline B_{1} & B_{2} \\
\hline
\end{array} \longmapsto \begin{array}{|c|c|}
\hline U & q V \\
\hline-V & U-p V \\
\hline
\end{array} \cdot \begin{array}{|c|c|}
\hline A_{1} & A_{2} \\
\hline B_{1} & B_{2} \\
\hline
\end{array} \cdot \begin{array}{|c|c|c|}
\hline Z-p T & -q T \\
\hline T & Z \\
\hline
\end{array}
$$


To each $n \times n \mathbb{G}$-matrix $U+\xi V$ corresponds a formally complex matrix \begin{tabular}{|l|l|}
\hline$U-p V$ & $-q V$ \\
\hline$V$ & $U$ \\
\hline
\end{tabular} dence induces an isomorphism between the ring of $n \times n$ matrices over $\mathbb{G}$ and the ring of $2 n \times 2 n$ formally complex matrices.

Notice that formally complex matrix associated to $\overline{U+\xi V}$ has the \begin{tabular}{|c|c|}
\hline$U$ & $q V$ \\
\hline & form \\
\hline
\end{tabular} follows

$$
\begin{array}{|c|c|}
\hline A_{1} & A_{2} \\
\hline B_{1} & B_{2} \\
\hline
\end{array} \longmapsto \begin{array}{|c|c|c|}
\hline U-p V & -q V \\
\hline V & U \\
\hline A_{1} & A_{2} \\
\hline B_{1} & B_{2} \\
\hline
\end{array} \cdot \begin{array}{|c|c|c|}
\hline Z-p T & -q T \\
\hline T & Z \\
\hline
\end{array} .
$$

4.3.1. The separable case. First case: char $\mathbb{G} \neq 2$. Assume that $\mathbb{F} \subset \mathbb{F}(\xi)=\mathbb{G}$ is a separable extension, then we can consider the minimal polynomial $\wp(t)=t^{2}+q$, in this case the transformation (4.5) is turned into

$$
\begin{array}{|c|c|}
\hline A_{1} & A_{2} \\
\hline B_{1} & B_{2} \\
\hline
\end{array}
$$

Precisely, the transformation (4.6) corresponds to the homogeneous biquadratic problem which is reduced to the semilinear Kronecker problem, see [26].

Let $\mathbb{G}$ be a field with an automorphism $\sigma$, so, the semilinear Kronecker problem is to find matrix canonical forms for the pair $(A, B)$ of matrices over $\mathbb{G}$ of equal size, by the following transformation

$$
(A, B) \longmapsto\left(X^{-1} A Y, X^{-1} B Y^{\sigma}\right)
$$

Djoković solved this problem in [5] and the solution of the corresponding homogeneous biquadratic problem ([26], Theorem 3) induces the indecomposable matrix corepresentation $K_{8}-6_{i}$, see Section 6 for notations.

Remark 5. If we consider the canonical matrix form given in ([26], Theorem 4), the corepresentation type $K_{8}-6_{i}$ coincides with the corepresentation series of $K_{8}$ given in [20].

Second case: $\operatorname{char} \mathbb{G}=2$. Then, for the minimal polynomial $\wp(t)=t^{2}+p t+q$, it holds $p \neq 0$ and the transformation (4.4) takes 
the form

$$
\begin{array}{|c|c|}
\hline A_{1} & A_{2} \\
\hline B_{1} & B_{2} \\
\hline
\end{array} \longmapsto \begin{array}{|c|c|}
\hline U & -q V \\
\hline V & U-p V \\
\hline
\end{array} \cdot \begin{array}{|c|c|}
\hline A_{1} & A_{2} \\
\hline B_{1} & B_{2} \\
\hline
\end{array} \cdot \begin{array}{|c|c|c|}
\hline Z-p T & -q T \\
\hline T & Z \\
\hline
\end{array}
$$

this problem corresponds to the homogeneous biquadratic problem for a separable quadratic field extension $\mathbb{F} \subset \mathbb{G}$ with char $\mathbb{G}=2$ (see [27], Theorem 3). That induces the indecomposable matrix corepresentation $K_{8}-6_{i i}$.

4.3.2. The inseparable case. Now, consider an inseparable extension $\mathbb{F} \subset \mathbb{F}(\xi)=\mathbb{G}$, then char $\mathbb{G}=2$ and $p=0$, i.e., the minimal polynomial has the form $\wp(t)=t^{2}+q$, so (4.4) takes the form (4.6) which also corresponds to the homogeneous biquadratic problem considered in [27], it can be reduced to a $(\sigma, \delta)$-pseudolinear Kronecker problem solved in [21] by Sergeichuk, it deals with finding some matrix canonical forms for the pair of matrices $(A, B)$ over a field $\mathbb{G}$ of equal size by the following transformation

$$
(A, B) \longmapsto\left(X^{-1} A Y, X^{-1}\left(B Y^{\sigma}+A Y^{\delta}\right)\right)
$$

where $\sigma$ is an automorphism of $\mathbb{G}$ an $\delta$ is a right $\sigma$-derivation on $\mathbb{G}$ such that $(a b)^{\delta}=a b^{\delta}+a^{\delta} b^{\sigma}$. The solution of the homogeneous biquadratic problem associated is given in [27], Theorem 5, and these canonical form induces the corepresentation series $K_{8}-6_{i i i}$.

Now, we may check the described bijection in Theorem 3.7 and the values of the Tits form of the considered posets, Corollary 3.8.

\section{Proof of the Main Theorems}

\subsection{Theorem 3.1.}

Proof. Since both statements of the Theorem are self-dual, we proof only one of them, for instance (1). We breaking up the proof into four cases.

First case: $\mathcal{P}=K_{6}+(a \curlyvee b)$. Let $U \in$ Ind $\mathcal{P}$ be, we use induction on the number $\operatorname{dim} U_{0}$, when $\operatorname{dim} U_{0}=1$ the condition is satisfied trivially, one may inspect all indecomposables. Assume that $\operatorname{dim} U_{0}>$ 1 , and reasoning by contradiction, we suppose that $\widetilde{U}_{a} \neq U_{0}$ and $U_{a} \cap U_{b} \neq 0$. 
We consider $V=\left(V_{0}, V_{x}: x \in \mathcal{P}\right)$ such that $V_{0}=\tilde{U}_{a}$ and $V_{x}=$ $\tilde{U}_{a} \cap U_{x}$ and decompose it into a direct sum $V=\bigoplus_{i} V^{i}$, also consider $W=\left(W_{0}, W_{x}: x \in \mathcal{P}\right)$ where $V_{0} \oplus W_{0}=U_{0}$ and $W_{x}=\pi\left(V_{x}^{\prime}\right)$, where $V_{x}^{\prime}$ is some complement of $V_{x}$ in $U_{x}$ and $\pi$ is the natural projection.

Since $\operatorname{dim} V_{0}<\operatorname{dim} U_{0}$, every summand of $V$ satisfies the condition, then for some $i$, it holds $V_{a}^{i} \cap V_{b}^{i} \neq 0$ and $\widetilde{V}_{a}^{i}=\widetilde{V}_{b}^{i}=V_{0}^{i}$ (otherwise $\left.U_{a} \cap U_{b}=0\right)$. We claim that $V^{i}$ is a direct summand of $U$. A simple form to notice this is using matrix language. $M_{V_{b}^{i}}=11^{\eta_{1}} \oplus 1 \xi{ }^{\eta_{2}}$, however the second summand is trivially direct, then we may consider the following matrix, corresponding to the spaces $V_{b}^{i}$ and $W_{x}(x \in$ $\mathcal{P} \backslash a)$ :

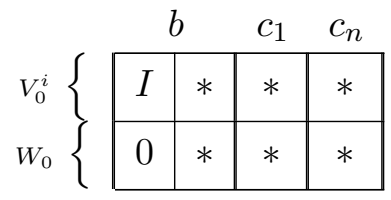

this matrix corepresentation is decomposed as a weak chain, see [20], Lemma 3.1, but additionally every space $W_{x}$ is not null, then the $I$ block, in (5.1), is a direct summand.

Second case: $\mathcal{P}=K_{6}+A^{-}+B^{-}$where $A^{-}=N(b) \backslash a^{\vee}$ and $B^{-}=N(a) \backslash b^{\vee}$. For this, we reason analogously to above case, but by considering the strong chain $B \triangleleft b$ instead of the weak one.

Third case: $\mathcal{P}=K_{6}+(a \curlyvee b)+A^{-}+B^{-}$, by using the above notations. We decompose the restriction $\left.U\right|_{K_{6}}=V=\bigoplus_{i} V^{i}$ into a direct sum of indecomposables, each of them satisfies first condition for $(a \curlyvee b)=\varnothing$, and thus second condition too, therefore we can decompose $V$ in a direct sum $X \oplus Y$, where $X_{a}+X_{b}=X_{0}$ and $\underset{\sim}{Y} a=\underset{\sim}{Y} b=0$, since $U$ is indecomposable then it is sincere either on some subset of $K_{6}+(a \curlyvee b)$ or on some subset of $K_{6}+A^{-}+B^{-}$.

Fourth case: $\mathcal{P}$ is any poset with a well inserted set $K_{6}$. We use induction on the number $|\mathcal{P} \backslash \mathcal{Q}|$ where $\mathcal{Q}=K_{6}+(a \curlyvee b)+A^{-}+B^{-}$. We consider an indecomposable corepresentation $U$ at all points of the subset $\mathcal{P} \backslash \mathcal{Q}$. First step, consider $\mathcal{P}=\mathcal{Q}$, this has been proved in the third case.

Now suppose $x \in \mathcal{P} \backslash \mathcal{Q}$, it may happen three cases: (a) $a \triangleleft x$ or (b) $b \triangleleft x$ or (c) $x<\{a, b\}$. The first two cases are analogous, for instance 
$a \triangleleft x$, we consider the restriction $V=\left.U\right|_{\mathcal{P} \backslash x}$ and decompose it in a direct sum $\bigoplus_{i} V^{i}$, each $V^{i}$ satisfies the condition (1) but $\tilde{V}_{a}^{i} \neq V_{0}^{i}$ for all $i$, otherwise $V^{i}$ would be indecomposable, then $V_{a}^{i} \cap V_{b}^{i}=0$ for every $i$, therefore it holds $V_{a} \cap V_{b}=0$ and $U_{a} \cap U_{b}=0$. For the case (c) we follow the same way, $\left.U\right|_{\mathcal{P} \backslash x}=V=\bigoplus_{i} V^{i}$, but $V_{a}^{i} \cap V_{b}^{i} \neq 0$ for all $i$, so that $U$ appears to be indecomposable, then $V_{a} \cap V_{b} \neq 0$ and therefore it holds $\widetilde{V}_{a}=\widetilde{V}_{b}=V_{0}$ and $\widetilde{U}_{a}=\widetilde{U}_{b}=U_{0}$.

We have proved the inductive step and that completes the proof of theorem 3.1.

Corollary 3.2 follows directly from Theorem 3.1.

\subsection{Theorem 3.3.}

Proof. The conditions (1) and (2) of this Theorem are mutually dual, we prove the first one by taking induction on the number $\operatorname{dim} U_{0}$. Let $\mathcal{P}$ be a poset with a well inserted subposet $K_{8}$ of the form (3.1).

First case: $\mathcal{P}=K_{8}+\Omega^{+}$. Let $U$ be an indecomposable corepresentation of $\mathcal{P}$, when $\operatorname{dim} U_{0}=1, \mathcal{P}$ satisfies the condition trivially. For an upper dimension $\operatorname{dim} U_{0}=n$, by reasoning for contradiction, we assume (a) $U_{r}+U_{s} \neq U_{0}$ and denote $V_{0}=U_{r}+U_{s}$. Consider the corepresentation $V=\left(V_{0}, V_{x}: x \in \mathcal{P}\right)$ by setting $V_{x}=U_{x} \cap V_{0}$ for $x \in \mathcal{P}$ and decompose it into a direct sum of indecomposables $V=\bigoplus_{i} V^{i}$.

Let $W_{0}, V_{x}^{\prime}$ be some complements for $V_{0}$ and $V_{x}$ in $U_{0}$ and $U_{x}$ respectively, so we obtain the corepresentation $W=\left(W_{0}, W_{x}: x \in\right.$ $\mathcal{P})$ with $W_{x}=\pi\left(V_{x}^{\prime}\right)$ where $\pi: U_{0} \rightarrow W_{0}$ is the natural projection.

For first part of the condition (1), we admit for example that $U_{a} \cap$ $U_{r} \neq 0$ and we choose a fixed index $i$ such that $V_{a}^{i} \cap V_{r}^{i} \neq 0$, then by induction hypothesis it follows $\tilde{V}_{a}^{i}=V_{r}^{i}+V_{s}^{i}=V_{0}^{i}$ and we can conclude that corepresentation $V^{i}$ is a direct summand of $U$. A form to notice this decomposition is via matrix considerations, for this, we reduce the matrix block associated to points in Supp $W \subset a+\Omega^{+}$, i.e., we reduce a matrix block associated either to the subposet $A_{38}^{*} \subset \mathcal{P}$ or to a weak chain with maximum length 3 . By applying right column additions from the blocks $M_{V^{i}}$ and right row additions from the blocks $M_{W}$, we annul the blocks in the intersection. 
Also, we can assume (b) $\widetilde{U}_{a} \neq U_{0}$, this time we set $V_{0}=\widetilde{U}_{a}$, and construct the corepresentation $V=\bigoplus_{i} V^{i}$, and $W$ analogously. By reasoning in similar way we find a direct summand $V^{i}$ of $V$ which is a direct summand of $U$, however, in this case the matrix consideration leads to support of $W$ be either the set $A_{28}^{*}$ or a subset of finite type of the set $\{r \triangleleft p \triangleleft q, s\}$ where $p$ and $q$ are weak points.

Second case: $\mathcal{P}=K_{8}+\Delta^{-}+\Gamma^{-}$. By reasoning as above, we may assume (a) $U_{r}+U_{s} \neq U_{0}$ (or (b) $\widetilde{U}_{a} \neq U_{0}$ ), so, we set $V_{0}=$ $U_{r}+U_{s}$ (or resp. $V_{0}=\widetilde{U}_{a}$ and considering the same construction for $V$ and $W$, we obtain a direct summand of $U$, however, the matrix calculations are more simply because Supp $W \subset\left\{\Delta^{-} \triangleleft a\right\}$ is a chain (or Supp $W \subset \Gamma^{-} \triangleleft\{r, s\}$ respectively).

Third case: $\mathcal{P}=K_{8}+\Omega^{+}+\Delta^{-}+\Gamma^{-}$. The support of any indecomposable corepresentation $U$ of $\mathcal{P}$ is $K_{8}+\Omega^{+}$or $K_{8}+\Delta^{-}+\Gamma^{-}$, and it returns us to the first or second case respectively. For this, decompose the restriction $V=\left.U\right|_{K_{8}}$ into a direct sum of indecomposables $V=\bigoplus_{i} V^{i}$, since each of them satisfies the condition (2), we get $V=X \oplus Y$ where $X_{a}+X_{r}=X_{a}+X_{s}=X_{0}$ and $\underset{\sim}{Y}=Y_{r} \cap Y_{s}=0$ therefore $U$ is sincere either on some subset of $K_{8}+\Omega^{+}$or on some subset of $K_{8}+\Delta^{-}+\Gamma^{-}$.

Fourth case: $\mathcal{P}$ is an arbitrary poset. Let $U \in \operatorname{Ind} \mathcal{P}$ be a sincere on the subposet $\mathcal{P} \backslash \mathcal{Q}$ where $\mathcal{Q}=K_{8}+\Omega^{+}+\Delta^{-}+\Gamma^{-}$, we proof the statement by induction on the number $|\mathcal{P} \backslash \mathcal{Q}|$, the base statement holds for $\mathcal{P}=Q$, it was proved in the previous cases.

For $x \in \mathcal{P} \backslash \mathcal{Q}$, we decompose the restriction $V=\left.U\right|_{\mathcal{P} \backslash x}$ into a direct sum $V=\bigoplus_{i} V^{i}$, we have four cases: (a) $a \triangleleft x$, (b) $\{r, s\} \triangleleft x$, (c) $x<\{r, a\}$ and (d) $x<\{s, a\}$. Since $U$ is sincere, for (a) it holds $\tilde{V}_{a}^{i} \neq V_{0}^{i}$ and for (b) it holds $V_{r}^{i}+V_{s}^{i} \neq V_{0}^{i}$ for all $i$, so, in any case we have $V_{a}^{i} \cap V_{r}^{i}=V_{a}^{i} \cap V_{s}^{i}=0$ for all $i$, therefore $U_{a} \cap U_{r}=U_{a} \cap U_{s}=0$. For (c) and (d) it holds $V_{a}^{i} \cap V_{r}^{i} \neq 0$ and $V_{a}^{i} \cap V_{s}^{i} \neq 0$ for all $i$ respectively, therefore $\widetilde{U}_{a}=U_{r}+U_{s}=U_{0}$.

The proof follows from the four previous cases.

Corollary 3.4 follows directly from Theorem 3.3. 


\section{Notations for Appendixes}

We consider an arbitrary quadratic field extension $\mathbb{F} \subset \mathbb{F}(\xi)=\mathbb{G}$ and minimal polynomial $\wp(t)=t^{2}+p t+q$ for the generator $\xi$, unless we say other wise.

In the whole appendixes we use the following notation for matrix corepresentation types. $I_{n}$ represents the identity matrix of $n \times n$ size. $\overleftarrow{A}$ (resp. $A^{\uparrow}$ ) is came from $A$ adding a zero column (row) on the left side (on the top), analogously are defined $\vec{A}, A^{\downarrow}$, even if $A$ has several arrows we add several zero columns or rows. $J_{n}(\lambda)$ is a Jordan block of order $n$ with eigenvalues $\lambda$, the identities can be written both under or above the diagonal, and $J_{n}^{+}(\lambda)$ (resp. $J_{n}^{-}(\lambda)$ ) means the Jordan block with the identities written only above (under) the diagonal.

For $P_{2}=\left(\begin{array}{ll}1 & \xi \\ 0 & 1\end{array}\right)$ and $P_{2}^{-}=\left(\begin{array}{ll}1 & 0 \\ \xi & 1\end{array}\right)$ set

$$
\widetilde{P}_{2 k}=P_{2 k}=\bigoplus_{i=1}^{k} P_{2} \quad P_{2 k}^{-}=\bigoplus_{i=1}^{k} P_{2}^{-}
$$

in particular, $P_{0}^{-}, P_{0}$ and $\widetilde{P}_{0}$ are formal matrices of one column and zero rows, also we define its odd parts

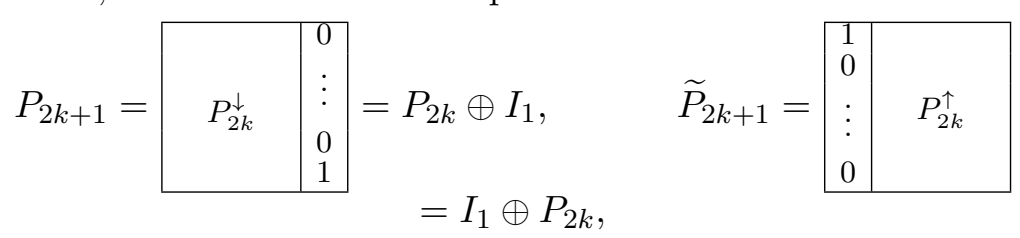

where $P_{1}=\widetilde{P}_{1}=I_{1}$.

Set $R_{2 k+2}=P_{2 k} \oplus 1 \xi, \quad R_{2 k+3}=I_{1} \oplus P_{2 k} \oplus 1 \xi \quad$ and $\quad C_{n}=$

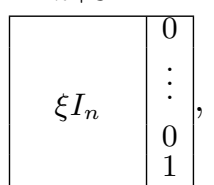

in particular, $R_{2}=1 \xi$ and $R_{3}=I_{1} \oplus 1 \xi$. Other notations are the following 


$$
\begin{array}{rlrl}
{ }^{+} Q_{2 k} & =Q_{2 k}^{+}=P_{2 k} \oplus P_{2 k}^{-} & Q_{2 k+1}^{+} & =P_{2 k+2} \oplus P_{2 k}^{-} \\
{ }^{-} Q_{2 k} & =P_{2 k}^{-} \oplus P_{2 k-2} \oplus R_{2} & { }^{+} Q_{2 k+1} & =P_{2 k} \oplus P_{2 k+2}^{-} \\
Q_{2 k}^{-} & =R_{2} \oplus P_{2 k-2}^{-} \oplus P_{2 k} & Q_{2 k+1} & =P_{2 k}^{-} \oplus P_{2 k} \oplus R_{2} \\
Q_{2 k+1}^{-} & =R_{2} \oplus P_{2 k}^{-} \oplus P_{2 k},
\end{array}
$$

the matrices ${ }^{+} Q_{0}, Q_{0}^{+},{ }^{+} \widetilde{Q}_{0}$ and $\widetilde{Q}_{0}^{+}$represent a matrix with one column and zero rows. By permuting the summands we obtain

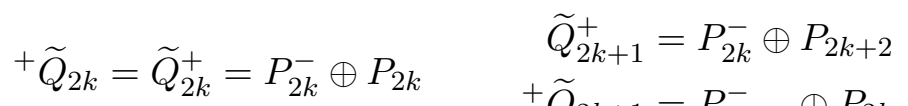

$$
\begin{aligned}
& -\widetilde{Q}_{2 k}=R_{2} \oplus P_{2 k-2} \oplus P_{2 k}^{-} \quad+\widetilde{Q}_{2 k+1}=P_{2 k+2}^{-} \oplus P_{2 k} \\
& \begin{array}{rlrl}
\widetilde{Q}_{2 k}^{-}=P_{2 k} \oplus P_{2 k-2}^{-} \oplus R_{2} & { }^{-} \widetilde{Q}_{2 k+1} & =R_{2} \oplus P_{2 k} \oplus P_{2 k}^{-} \\
\widetilde{Q}_{2 k+1}^{-} & =P_{2 k} \oplus P_{2 k}^{-} \oplus R_{2},
\end{array}
\end{aligned}
$$

Each poset $\mathcal{P}$ has associated an imaginary root $\mu$. A dimension vector $d$ with $d_{0}=0$ corresponds to matrix corepresentations with zero columns, so, we only have considered admissible roots with $d_{0}>$ 0 . The minimal dimension vector is denoted by dmin. Dimensions of each indecomposable corepresentation type form a sequence that starts at dmin and increase in a multiple of $\mu$, this increase is called step. The indecomposable $K_{6}-\widetilde{i}\left(\operatorname{resp} . K_{8}-\widetilde{j}\right.$ ) is obtained from $K_{6}-i$ $\left(K_{8}-j\right)$ by switching the point $a$ with $b(r$ with $s)$ and viceversa.

In Appendix A, for the type $K_{6}-6$ the matrix block $X$ is an indecomposable Frobenius block over $\mathbb{F}$.

In Appendix B we divide the classification of indecomposable corepresentations of $K_{8}$ in four parts, the first three follow the matrix model presented at the beginning of this appendix, the fourth part consists of one corepresentation type. Also, we consider the following notation for the corepresentation series. If $f(t)=a_{0}+a_{1} t+\cdots+a_{n-1} t^{n-1}+t^{n}$ is a monic non-constant polynomial over $\mathbb{G}$, denote by $C(f)$ its standard companion matrix of order $n$ having the form (the numbers 1 occupy the diagonal next to the main one) 


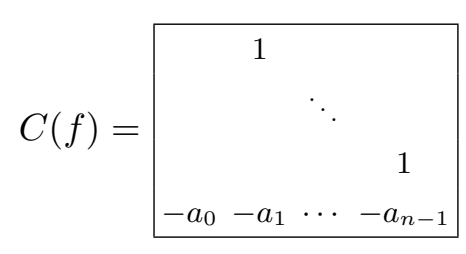

Let $\mathbb{G}[t, \sigma, \delta]$ be the skew polynomial ring consisting of right polynomials in the variable $t$ over $\mathbb{G}$ satisfying at $=t a^{\sigma}+a^{\delta}$ for any $a \in \mathbb{G}$, where $\sigma$ is an automorphism of $\mathbb{G}$ and $\delta$ is a $\sigma$-derivation on $\mathbb{G}$, see Section 4.3.2, and denote by $\mathcal{I}$ a maximal subset of pairwise non-similar indecomposable polynomials in $\mathbb{G}[t, \sigma, \delta]$.

The corepresentation series of $K_{8}$ for a separable quadratic field extension $\mathbb{F}=\mathbb{F}(\xi) \subset \mathbb{G}$ with char $\mathbb{G} \neq 2$ is given by $K_{8}-6_{i}$, so, we consider the minimal polynomial $\wp(t)=t^{2}-q$ and the natural conjugation $\overline{a+\xi b}=a-\xi b(a, b \in \mathbb{F})$. For $K_{8}-6_{i}$, the polynomial $f \in \mathcal{I}$ in $\mathbb{G}[t, \sigma, \delta]$ by setting the automorphism $\sigma(a+\xi b)=a-\xi b$ and $\delta=0$.

The corepresentation series of $K_{8}$ for a separable quadratic field extension with $\operatorname{char} \mathbb{G}=2$ is given by $K_{8}-6_{i i}$, then the minimal polynomial of $\xi$ is $\wp(t)=t^{2}+p t+q$ with $p \neq 0$. In this case the natural conjugation is given by $\overline{a+\xi b}=a-p b+\xi b$ and the polynomial $f \in \mathcal{I}$ in $\mathbb{G}[t, \sigma, 0]$ with $\sigma$ the natural conjugation.

The corepresentation series of $K_{8}$ for an inseparable extension has the form $K_{8}-6_{i i i}$, then char $\mathbb{G}=2$ and the minimal polynomial of $\xi$ is $\wp(t)=t^{2}+q$. For the series of this type, we take $f \in \mathcal{I}$ in $\mathbb{G}[t, \sigma, \delta]$ where $\sigma$ is the identity automorphism 1 and $\delta$ is the 1-derivation on $\mathbb{G}$ such that $\delta(a)=0(a \in \mathbb{F})$ and $\delta(\xi)=\xi$.

In Appendix $\mathrm{E}$ are presented the minimal roots of $K_{6}, K_{8}, A_{25}, A_{28}$, $A_{38}, A_{41}$ and their duals accompanied by the respective reflections of which they came from. Each of roots can be obtained through many reflections, in different forms. The number of compositions of reflections presented is minimal. Denote by $e_{x}$ the simple root associated to the point $x \in \mathcal{P}^{\bullet}$ and we name the above posets as in Figure 2 and Figure 4 . The reflection in the point $x \in \mathcal{P}^{\bullet}$ is denoted by $\rho_{x}$. The notation for the composition of reflection is the following

$$
\rho_{z}\left(\rho_{y}\left(e_{x}\right)\right)=\rho_{z} \rho_{y} e_{x}
$$


Appendix A. Classification of indecomposable corepresentations of the critical poset $K_{6}$

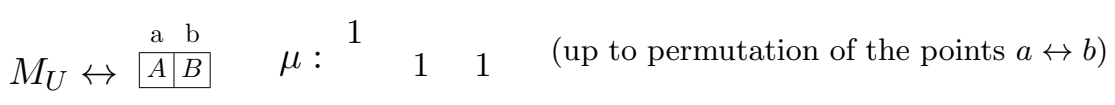

\begin{tabular}{|c|ccc|c|c|c|c|}
\hline type & \multicolumn{2}{|c|}{ dmin } & step & $f(d)$ & $A$ & $B$ \\
\hline 1 & 1 & 1 & 2 & $\mu$ & 1 & $\widetilde{P}_{n}$ & $R_{n+1}$ \\
\hline $1^{*}$ & 1 & 1 & 0 & $\mu$ & 1 & $P_{n}$ & $P_{n-1}^{\uparrow}$ \\
\hline 2 & 1 & 2 & 2 & $2 \mu$ & 2 & $-Q_{n}$ & $Q_{n}^{-}$ \\
\hline $2^{*}$ & 1 & 0 & 0 & $2 \mu$ & 2 & $Q_{n}^{+\downarrow}$ & $+Q_{n}^{\uparrow}$ \\
\hline $3=\widetilde{3}^{*}$ & 1 & 2 & 0 & $2 \mu$ & 2 & $R_{2 n+2}$ & $P_{2 n}^{\uparrow}$ \\
\hline $4=4^{*}$ & 2 & 2 & 2 & $2 \mu$ & 0 & $P_{2 n}$ & $R_{2 n}^{\uparrow}$ \\
\hline $5=5^{*}$ & 1 & 1 & 1 & $\mu$ & 0 & $I_{n}$ & $I_{n}+\xi J_{n}^{+}(0)$ \\
\hline $6=6^{*}$ & 1 & 1 & 1 & $\mu$ & 0 & $I_{n}$ & $\xi I_{n}+X$ \\
\hline
\end{tabular}

Appendix B. Classification of indecomposable corepresentations of the critical poset $K_{8}$
(i) $\quad M_{U} \leftrightarrow \quad$\begin{tabular}{c|c|cc}
$\mathrm{r}$ & $\mathrm{s}$ & $\mathrm{a}$ \\
$R$ & $A_{1}$ & $A_{2}$ \\
$S$ & $A_{1}^{\prime}$ & $A_{2}^{\prime}$
\end{tabular}$\quad \quad$ (up to permutation of the points $r \leftrightarrow s$ )
(ii) $\quad M_{U} \leftrightarrow$\begin{tabular}{|c|c|cc|}
$\mathrm{r}$ & $\mathrm{s}$ & \multicolumn{2}{c}{$\mathrm{a}$} \\
\hline $10 \ldots 0$ & $10 \ldots$ & $T_{1}$ & $T_{2}$ \\
\hline$R$ & & $A_{1}$ & $A_{2}$ \\
& $S$ & $A_{1}^{\prime}$ & $A_{2}^{\prime}$ \\
\hline
\end{tabular}$\quad \quad \mu:$\begin{tabular}{llll}
2 & & & \\
\hline
\end{tabular}

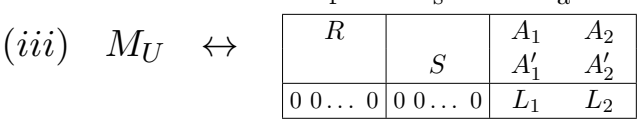


Part $(i)$

\begin{tabular}{|c|c|c|c|c|c|c|c|c|c|}
\hline type & dmin & step & $f(d)$ & $R$ & $A_{1}$ & $A_{2}$ & $S$ & $A_{1}^{\prime}$ & $A_{2}^{\prime}$ \\
\hline 1 & $\begin{array}{llll}2 & & & \\
& 1 & 1 & 3 \\
\end{array}$ & $\mu$ & 1 & $I_{n}$ & $\overrightarrow{I_{n}}$ & $\xi I_{n}$ & $I_{n}$ & $\overleftarrow{I_{n}}$ & $\xi I_{n}$ \\
\hline $1^{*}$ & $\begin{array}{llll}2 & & & \\
& 1 & 1 & 1\end{array}$ & $\mu$ & 1 & $I_{n+1}$ & $I_{n}^{\downarrow}$ & $\xi I_{n+1}$ & $I_{n+1}$ & $I_{n}^{\uparrow}$ & $\xi I_{n+1}$ \\
\hline $2=\tilde{2}^{*}$ & $\begin{array}{llll}1 & & & \\
& 1 & 0 & 1\end{array}$ & $\mu$ & 1 & $I_{n+1}$ & $I_{n+1}$ & $\xi I_{n}^{\uparrow}$ & $I_{n}$ & $\overrightarrow{I_{n}}$ & $\xi I_{n}$ \\
\hline 3 & $\begin{array}{llll} & & & \\
& 1 & 0 & 2 \\
\end{array}$ & $\mu$ & 2 & $I_{n+1}$ & $I_{n+1}$ & $\xi I_{n+1}$ & $I_{n}$ & $\overrightarrow{I_{n}}$ & $\xi \overleftarrow{I_{n}}$ \\
\hline $3^{*}$ & $\begin{array}{lllll} & & & & \\
& 0 & 1 & 0 \\
\end{array}$ & $\mu$ & 2 & $I_{n}$ & $I_{n}$ & $\xi I_{n}$ & $I_{n+1}$ & $I_{n}^{\uparrow}$ & $\xi I_{n}^{\downarrow}$ \\
\hline $4=4^{*}$ & $\begin{array}{llll}2 & 1 & 1 & 2 \\
& & 1 & 1 \\
\end{array}$ & $\mu$ & 0 & $I_{n}$ & $I_{n}$ & $\xi J_{n}(0)$ & $I_{n}$ & $I_{n}$ & $\xi I_{n}$ \\
\hline $5=5^{*}$ & $\begin{array}{llll}2 & 1 & 1 & 2 \\
& & 1 & 1 \\
\end{array}$ & $\mu$ & 0 & $I_{n}$ & $I_{n}$ & $\xi J_{n}(1)$ & $I_{n}$ & $I_{n}$ & $\xi I_{n}$ \\
\hline $6_{i}$ & $\begin{array}{llll}2 & & 1 & \\
& & 1 & 2 \\
\end{array}$ & $\mu$ & 0 & $I_{n}$ & $I_{n}$ & $\xi I_{n}$ & $I_{n}$ & {$\left[I_{n}-\overline{C(f)}\right]$} & $\xi\left[I_{n}+\overline{C(f)}\right]$ \\
\hline $6_{i i}$ & $\begin{array}{llll}2 & 1 & 1 & 2 \\
& & 1\end{array}$ & $\mu$ & 0 & $I_{n}$ & $I_{n}$ & $\xi I_{n}$ & $I_{n}$ & {$\left[I_{n}+\overline{C(f)}\right]$} & {$\left[\bar{\xi} I_{n}+\xi \overline{C(f)}\right]$} \\
\hline $6_{i i i}$ & $\begin{array}{lllll} & 2 & & & \\
& 1 & 1 & 2\end{array}$ & $\mu$ & 0 & $I_{n}$ & $I_{n}$ & $\xi I_{n}$ & $I_{n}$ & {$[\overline{C(f)}]$} & $\xi\left[I_{n}+C(f)\right]$ \\
\hline
\end{tabular}

Part (ii)

\begin{tabular}{|c|c|c|c|c|c|c|c|c|c|c|c|}
\hline type & dmin & step & $f(d)$ & $R$ & $A_{1}$ & $A_{2}$ & $S$ & $A_{1}^{\prime}$ & $A_{2}^{\prime}$ & $T_{1}$ & $T_{2}$ \\
\hline 7 & $\begin{array}{lll}1 & 1 & 1\end{array}$ & $\mu$ & 1 & $\overleftarrow{I_{n}}$ & $\overrightarrow{I_{n}}$ & $\xi I_{n}$ & $\overleftarrow{I_{n}}$ & $\overleftarrow{I_{n}}$ & $\xi I_{n}$ & $\mid \begin{array}{llll}0 & 0 & \ldots & 0\end{array}$ & $\begin{array}{llll}1 & 0 & \ldots & 0\end{array}$ \\
\hline 8 & $\begin{array}{lll}1 & 1 & 2\end{array}$ & $\mu$ & 2 & $\overleftarrow{I_{n}}$ & $\overrightarrow{I_{n}}$ & $\xi \overleftarrow{I_{n}}$ & $\overleftarrow{I_{n}}$ & $\overleftarrow{I_{n}}$ & $\xi \overrightarrow{I_{n}}$ & $\begin{array}{llll}0 & \ldots & 0 & 1\end{array}$ & $\begin{array}{llll}0 & \ldots & 0 & \xi\end{array}$ \\
\hline 9 & $\begin{array}{lll}122 \\
\end{array}$ & $\mu$ & 2 & $\overleftarrow{I_{n}}$ & $\overrightarrow{I_{n}}$ & $\xi \overleftarrow{I_{n}}$ & $\longdiv { I _ { n + 1 } }$ & $I_{n+1}$ & $\xi I_{n+1}$ & .0 & 10. \\
\hline 10 & $\begin{array}{lll}1 & 1 & 0\end{array}$ & $\mu$ & 2 & $\overleftarrow{I_{n}}$ & $I_{n}$ & $\xi J_{n}(1)$ & $\overleftarrow{I_{n}}$ & $I_{n}$ & $\xi I_{n}$ & $\begin{array}{llll}0 & 0 & \ldots & 0\end{array}$ & $\begin{array}{llll}1 & 0 & \ldots & 0\end{array}$ \\
\hline
\end{tabular}

Part (iii)

\begin{tabular}{|c|c|c|c|c|c|c|c|c|c|c|c|}
\hline pe & $\min$ & ster & $f(d)$ & $R$ & $A_{1}$ & $A_{2}$ & $S$ & $A_{1}^{\prime}$ & $4_{2}^{\prime}$ & $L_{1}$ & $L_{2}$ \\
\hline $7^{*}$ & $\begin{array}{lll}0 & 0 & 1 \\
\end{array}$ & $\mu$ & 1 & $x_{n}$ & & & & $I_{n}$ & & & \\
\hline $8^{*}$ & $\begin{array}{lll}0 & 0 & 0 \\
\end{array}$ & 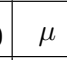 & 2 & & & & & & 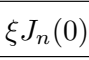 & & \\
\hline 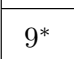 & 102 & $\beta_{0}$ & 2 & & & & & & & $\ldots 11$ & $\xi$ \\
\hline $10^{*}$ & $0 \quad 0$ & $\mu$ & 2 & & & & & & $\vec{s} \overrightarrow{ }$ & $\ldots 01$ & 0 \\
\hline
\end{tabular}

São Paulo J.Math.Sci. 4, 2 (2010), 141-175 
Part $(i v)$

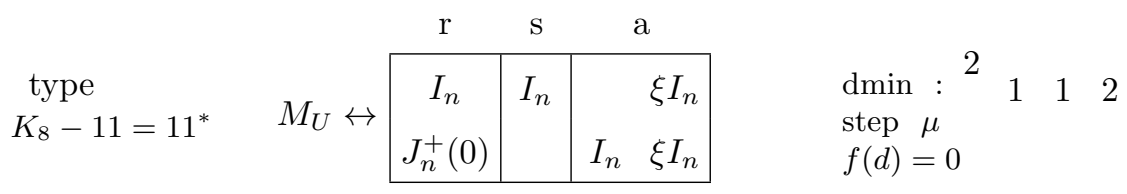

Appendix C. Classification of indecomposable corepresentations of the posets $A_{25}$ and $A_{25}^{*}$

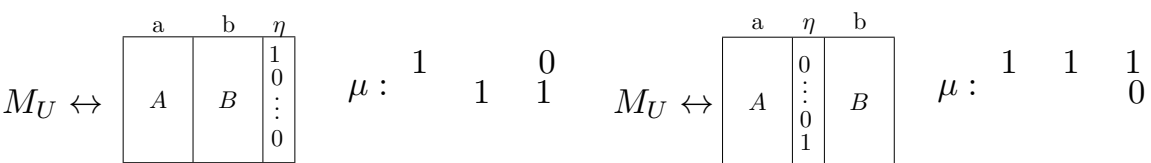

\begin{tabular}{|c|ccr|c|c|c|c|}
\hline type & \multicolumn{2}{|c|}{ dmin } & step & $f(d)$ & $A$ & $B$ \\
\hline 1 & 1 & 1 & 0 & $\mu$ & 1 & $P_{n}$ & $P_{n-1}^{\uparrow}$ \\
\hline 2 & 2 & 2 & 0 & $2 \mu$ & 2 & $+Q_{n}$ & $+Q_{n-1}^{\uparrow \downarrow}$ \\
\hline 3 & 1 & 0 & 0 & $2 \mu$ & 2 & $Q_{n}^{+\downarrow}$ & $+Q_{n}^{\uparrow}$ \\
\hline 4 & 1 & 2 & 0 & $2 \mu$ & 2 & $R_{2 n+2}$ & $P_{2 n}^{\uparrow}$ \\
\hline 5 & 2 & 2 & 1 & $2 \mu$ & 2 & $P_{2 n}$ & $R_{2 n}^{\uparrow}$ \\
\hline
\end{tabular}

\begin{tabular}{|c|ccr|c|c|c|c|}
\hline type & \multicolumn{3}{|c|}{ dmin } & step & $f(d)$ & $A$ & $B$ \\
\hline 1 & 1 & 1 & 0 & $\mu$ & 1 & $\widetilde{P}_{n}$ & $\widetilde{P}_{n-1}^{\downarrow}$ \\
\hline 2 & 2 & 2 & 2 & $2 \mu$ & 2 & $\widetilde{Q}_{n}^{+}$ & $Q_{n}^{-\downarrow}$ \\
\hline 3 & 1 & 2 & 0 & $2 \mu$ & 2 & $Q_{n}^{-}$ & $+\widetilde{Q}_{n-1}^{\downarrow}$ \\
\hline 4 & 1 & 0 & 0 & $2 \mu$ & 2 & $P_{2 n}^{\uparrow}$ & $P_{2 n}^{\downarrow}$ \\
\hline 5 & 2 & 2 & 0 & $2 \mu$ & 2 & $P_{2 n}$ & $P_{2 n-2}^{\uparrow \downarrow}$ \\
\hline
\end{tabular}

Appendix D. Classification of indecomposable corepresentations of the posets $A_{38}$ and $A_{38}^{*}$

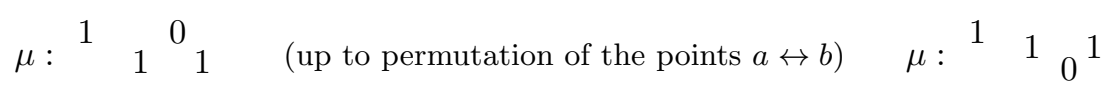




\begin{tabular}{|c|c|c|c|c|c|c|}
\hline type & dmin & step & $f(d)$ & $A$ & $B$ & $P$ \\
\hline 1 & $\begin{array}{lll}1 & & 1 \\
& 0 & 0\end{array}$ & $\mu$ & 1 & $I_{n}^{\downarrow}$ & $I_{n}^{\downarrow}+C_{n-1}^{\uparrow}$ & $\begin{array}{c}0 \\
\xi \\
\vdots \\
\vdots\end{array}$ \\
\hline 2 & $\begin{array}{lll}1 & & 1 \\
& 0 & 1\end{array}$ & $\mu$ & 1 & \begin{tabular}{l|l}
$I_{n}^{\downarrow}$ & $I_{r}$
\end{tabular} & $I_{n+1}+\xi J_{n+1}^{-}(0)$ & $\begin{array}{c}0 \\
\vdots\end{array}$ \\
\hline 3 & $\begin{array}{lll}1 & & 1 \\
& 1 & 1\end{array}$ & $\mu$ & 1 & $I_{n}$ & $I_{n}+\xi J_{n}^{-}(0)$ & $\begin{array}{c}0 \\
\vdots\end{array}$ \\
\hline 4 & $\begin{array}{lll}1 & & 2 \\
& 0 & 0\end{array}$ & $2 \mu$ & 2 & $I_{2 n}^{\downarrow}$ & $I_{2 n}^{\downarrow}+C_{2 n-1}^{\uparrow}$ & $\begin{array}{c}\xi 0 \\
0 \xi \\
\vdots \vdots\end{array}$ \\
\hline type & dmin & step & $f(d)$ & $\overline{A A}$ & $\bar{B}$ & $\bar{Q}$ \\
\hline 1 & $\begin{array}{llll}1 & & & \\
& & & 1\end{array}$ & $\mu$ & 1 & $C_{n}^{\uparrow}$ & $C_{n}^{\uparrow}+J_{n+1}^{+}(0)$ & \\
\hline 2 & $\begin{array}{lll}1 & 1 & \\
& & 1\end{array}$ & $\mu$ & 1 & $C_{n}^{\uparrow}$ & $I_{n}^{\downarrow}+\xi I_{n}^{\uparrow}$ & $\vdots$ \\
\hline 3 & 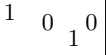 & $\mu$ & 1 & $\xi I_{n}^{\uparrow}$ & $\xi I_{n}^{\uparrow}+I_{n}^{\downarrow}$ & : \\
\hline 4 & $\begin{array}{lll}1 & 0 & \\
& & \\
& 0\end{array}$ & $2 \mu$ & 2 & $C_{2 n-1}^{\Uparrow \uparrow}$ & $1 \quad C_{2 n-1}^{\Uparrow \uparrow}+I_{2 n}^{\downarrow}$ & $\begin{array}{c}0 \xi \\
\vdots \vdots \\
0\end{array}$ \\
\hline
\end{tabular}

\section{Appendix E. Roots and Reflections}

\begin{tabular}{|c|c|c|c|}
\hline \multicolumn{4}{|c|}{$A_{25}$} \\
\hline Type & $\left(d_{0} ; d_{a} d_{b} d_{\eta}\right)$ & $\mathrm{f}$ & reflections \\
\hline 1 & 11101 & 1 & $\rho_{\eta} \rho_{0} e_{a}$ \\
\hline 2 & 2201 & 2 & $\rho_{0} \rho_{\eta} \rho_{a} e_{0}$ \\
\hline 3 & $\begin{array}{llll}1 & 0 & 0 & 1\end{array}$ & 2 & $\rho_{\eta} e_{0}$ \\
\hline 4 & 1201 & 2 & $\rho_{\eta} \rho_{a} e_{0}$ \\
\hline 5 & 2221 & 2 & $\rho_{b} \rho_{0} \rho_{\eta} \rho_{a} e_{0}$ \\
\hline
\end{tabular}

\begin{tabular}{|c|c|c|c|}
\hline \multicolumn{4}{|c|}{$A_{25}^{*}$} \\
\hline Type & $\left(d_{0} ; d_{a} d_{\eta} d_{b}\right)$ & $\mathrm{f}$ & reflections \\
\hline 1 & 1110 & 1 & $\rho_{\eta} \rho_{0} e_{a}$ \\
\hline 2 & 2212 & 2 & $\rho_{b} \rho_{0} \rho_{\eta} \rho_{a} e_{0}$ \\
\hline 3 & 1210 & 2 & $\rho_{\eta} \rho_{a} e_{0}$ \\
\hline 4 & 1010 & 2 & $\rho_{\eta} e_{0}$ \\
\hline 5 & 2210 & 2 & $\rho_{0} \rho_{\eta} \rho_{a} e_{0}$ \\
\hline
\end{tabular}

São Paulo J.Math.Sci. 4, 2 (2010), 141-175 


\begin{tabular}{|l|c|r|r|}
\hline \multicolumn{5}{|c|}{$A_{38}$} \\
\hline Type & $\left(d_{0} ; d_{a} d_{b} d_{p}\right)$ & $\mathrm{f}$ & reflections \\
\hline 1 & 1001 & 1 & $\rho_{0} e_{p}$ \\
2 & 1011 & 1 & $\rho_{p} \rho_{0} e_{b}$ \\
3 & 1111 & 1 & $\rho_{b} \rho_{p} \rho_{0} e_{a}$ \\
4 & 1002 & 2 & $\rho_{p} e_{0}$ \\
\hline \multicolumn{5}{|c|}{$K_{6}$} \\
\hline Type & $\left(d_{0} ; d_{a} d_{b}\right)$ & $\mathrm{f}$ & reflections \\
\hline 1 & 112 & 1 & $\rho_{b} \rho_{0} e_{a}$ \\
$\widetilde{1}^{*}$ & 101 & 1 & $\rho_{0} e_{b}$ \\
2 & 122 & 2 & $\rho_{b} \rho_{a} e_{0}$ \\
$2^{*}$ & 100 & 2 & $e_{0}$ \\
3 & 120 & 2 & $\rho_{a} e_{0}$ \\
\hline
\end{tabular}

\begin{tabular}{|c|c|c|c|}
\hline \multicolumn{4}{|c|}{$A_{38}^{*}$} \\
\hline Type & $\left(d_{0} ; d_{q} d_{a} d_{b}\right)$ & $\mathrm{f}$ & reflections \\
\hline 1 & 1111 & 1 & $\overline{\rho_{b} \rho_{a} \rho_{0} e_{q}}$ \\
\hline 2 & 1110 & 1 & $\rho_{a} \rho_{0} e_{q}$ \\
\hline 3 & 1100 & 1 & $\rho_{0} e_{q}$ \\
\hline 4 & 1200 & 2 & $\rho_{q} e_{0}$ \\
\hline \multicolumn{4}{|c|}{$K_{8}$} \\
\hline Type & $\left(d_{0} ; d_{r} d_{s} d_{a}\right)$ & $\mathrm{f}$ & reflections \\
\hline 1 & 2113 & 1 & $\rho_{a} r_{0} \rho_{s} \rho_{r} r_{0} e_{a}$ \\
\hline$\widetilde{1}^{*}$ & 2111 & 1 & $r_{0} \rho_{s} \rho_{r} r_{0} e_{a}$ \\
\hline 2 & 1101 & 1 & $\rho_{r} r_{0} e_{a}$ \\
\hline 3 & 1102 & 2 & $\rho_{a} \rho_{r} e_{0}$ \\
\hline $3^{*}$ & 1010 & 2 & $\rho_{s} e_{0}$ \\
\hline 7 & 1111 & 1 & $\rho_{s} \rho_{r} r_{0} e_{a}$ \\
\hline $7^{*}$ & 1001 & 1 & $r_{0} e_{a}$ \\
\hline 8 & 1112 & 2 & $\rho_{a} r_{2} \rho_{r} e_{0}$ \\
\hline $8^{*}$ & 1000 & 2 & $e_{0}$ \\
\hline 9 & 2122 & 2 & $\rho_{s} r_{0} \rho_{a} \rho_{r} e_{0}$ \\
\hline $9^{*}$ & 2102 & 2 & $r_{0} \rho_{a} \rho_{r} e_{0}$ \\
\hline 10 & 1110 & 2 & $\rho_{s} \rho_{r} e_{0}$ \\
\hline $10^{*}$ & 1002 & 2 & $\rho_{a} e_{0}$ \\
\hline
\end{tabular}

\begin{tabular}{|l|c|c|r|}
\hline \multicolumn{4}{|c|}{$A_{41}$} \\
\hline Type & $\left(d_{0} ; d_{q} d_{a} d_{b} d_{p}\right)$ & f & reflections \\
\hline 1 & 10101 & 1 & $\rho_{p} \rho_{0} e_{q}$ \\
\hline
\end{tabular}

\section{References}

[1] K. I. Belousov, L. A. Nazarova, A. V. Roiter \& V. V. SergEYCHUK, Elementary and multi-elementary representations of vectoroids (in Russian), Ukr. Math. J., 47:11 (1995), 1661-1687. English transl. in: Ukr. Mat. Zh., 47:11 (1995), 1451-1477.

[2] V.M. Bondarenko, A.G. ZavadskiJ, L.A. Nazarova, On representations of tame partially ordered sets, Representations and Quadratic Forms(in Russian), Inst. Mat. AN UkSSR, Kiev, (1979), 75106.English transl. in: Amer. Math. Soc. Transl.,(2)128(1986), 5578.

[3] Dieter Bünermann, Auslander-Reiten Köcher von einparametrigen teilweise geordneten Mengen, Dissertation, Düsseldorf, (1981).

[4] P. M. Cohn, Free ideal rings and localization in general rings, Cambridge university press, (2006).

[5] D.Ž. Duoković, Classification of pair consisting of a linear and semilinear map, Linear Algebra Appl., 20, (1978), 147-165.

[6] V. Dlab \& C. M. Ringel, Indecomposable representations of graphs and algebras, Memoirs Amer. Math. Soc., 173, (1976), 1-57. 
[7] V. Dlab \& C. M. Ringel, Normal forms of real matrices with respect to complex similarity, Linear Algebra Appl., 17, (1977), 107124.

[8] V. Dlab \& C. M. Ringel, Real subspaces of a quaternion vector space, Canad. J. Math., 30No.6, (1978), 1228-1242.

[9] P. Gabriel \& A. V. Roiter, Representations of FiniteDimensional Algebras, Algebra VIII, Encyclopedia of Math. Sc., 73 (1992).

[10] M. M. Kleiner, Partially ordered sets of finite type, Zap. Nauchn. Semin. LOMI (in Russian), 28 (1972), 32-41; English transl. in: J. Sov. Math., 3 (1975), 607-615.

[11] M. M. KLEINER, On faithful representations of partially ordered sets of finite type, Zap. Nauchn. Semin. LOMI (in Russian), 28 (1972), 42-59; English transl. in: J. Sov. Math., 3 (1975), 616-628.

[12] B. KlemP \& D. Simson, Schurian sp-representation-finite right peak PI-rings and their indecomposable socle projective modules, J. Algebra, 134:2, (1990), 390-468.

[13] L. KRONECKER, Algebraische Reduction der Scharen bilinearen Formen, Sitzungsber. Akad. Berlin, Jbuch. 22, 169 (1890), 1225-1237.

[14] L. A. Nazarova \& A. V. RoITER, Representations of partially ordered sets, Zap. Nauchn. Semin. LOMI (in Russian), 28 (1972), 5-31; English transl. in: J. Sov. Math., 3 (1975), 585-606.

[15] L. A. Nazarova \& A. V. RoITer, Polyquivers and Dinkin schemes, Funkts. Anal. Prilozh. (in Russian), 7, No.3, (1973), 9495; English transl. in: Funct. Anal. Apppl.,7 (1973), 252-253.

[16] L. A. Nazarova \& S.A. Ovsienko \& A. V. Roiter, Polyquivers of finite type, tr. Mat. Inst. Akad. Nauk SSSR 148, (1977).

[17] L. A. Nazarova, Representations of polyquivers of tame type, J. Sov. Math. 20, (1982), 2632-2656.

[18] L. A. Nazarova \& A. V. Roiter, Finitely represented dyadic sets, Ukr. Math. J., 52 No.10, (2000), 1561-1596.

[19] V.V. OTRASHEVSKAYA, One-parameter partially ordered sets (English. Russian original), Ukr. Math. J., v.28, (1976), 259-264.

[20] C. Rodríguez \& A.G. Zavadskis, On corepresentations of equipped posets and their differentiation, Revista Colombiana de Matemáticas, v.41, (2007), 117-142. Available in: http://www.emis.de/journals/RCM/Articulos/820.pdf

[21] V.V. SERGeichuk, Pseudolinear matrix pencils and systems of linear differential equations with meromorphic coefficients (in Russian), Differ. Uravneniya 25, (1989), 1721-1727. (English trans., Differ.Equations 25, (1989), 1201-1206)

[22] A. V. ZABARILO \& A. G. ZAVADSKIJ, Representations of oneparameter equipped posets, I, II, Matematychni Studii (in Russian), 
11 (1999), No. 1, 3-16 and No. 2, 119-134; Unabridge version in: Preprint 98.1 (in Russian), KSTUCA, Kiev (1998), 1-44 .

[23] A. G. ZavadskiJ, Tame equipped posets, Linear Algebra and its Appl., 365 (2003), 389-465.

[24] A. G. ZavadskiJ, Equipped posets of finite growth, Representations of Algebras and Related Topics, AMS, Fields Inst. Comm. Ser., 45 (2005), 363-396.

[25] A. G. ZAVADSKIJ, On two-point differentiation and its generalization, Algebraic Structures and Their Representations, AMS, Contemporary Math. Ser., 376 (2005), 413-436.

[26] A. G. ZAVADSKIJ, On the Kronecker problem and related problems of linear algebra, Linear Algebra and its Appl., 425 (2007), 26-62.

[27] A. G. ZavadskiJ, A matrix problem over a central quadratic skew field extension, Linear Algebra and its Appl., 428 (2008), 393-399.

[28] ZavadskiJ A.G., Nazarova L.A., Tame partially ordered sets, Matrix problems, Kiev, (1977), 122-143. 